\title{
RADIAL DISTRIBUTION OF ISM GAS-PHASE METALLICITY IN CLASH CLUSTERS AT $z \sim 0.35$ A NEW OUTLOOK ON ENVIRONMENTAL IMPACT ON GALAXY EVOLUTION
}

\author{
Anshu Gupta ${ }^{1}$, Tiantian Yuan ${ }^{1}$, Kim-Vy H. Tran ${ }^{2}$, Davide Martizzi ${ }^{3}$, Philip Taylor ${ }^{1}$, and Lisa J. Kewley ${ }^{1}$ \\ ${ }^{1}$ Research School of Astronomy and Astrophysics, Australian National University, Canberra, ACT 2611, Australia; anshu.gupta@anu.edu.au \\ ${ }^{2}$ George P. and Cynthia W. Mitchell Institute for Fundamental Physics and Astronomy, Department of Physics \& Astronomy, Texas A\&M University, \\ College Station, TX 77843, USA \\ ${ }^{3}$ Department of Astronomy, University of California, Berkeley, CA 94720-3411, USA \\ Received 2016 April 12; revised 2016 August 14; accepted 2016 August 21; published 2016 October 31
}

\begin{abstract}
We present the first observation of cluster-scale radial metallicity gradients from star-forming galaxies. We use DEIMOS on the Keck II telescope to observe two CLASH clusters at $z \sim 0.35$ : MACS J1115+0129 and RX $\mathrm{J} 1532+3021$. Based on our measured interstellar medium properties of star-forming galaxies out to a radius of $2.5 \mathrm{Mpc}$ from the cluster center, we find that the galaxy metallicity decreases as a function of projected clustercentric distance $(-0.15 \pm 0.08 \mathrm{dex} / \mathrm{Mpc})$ in MACS $1115+01$. On the mass-metallicity relation (MZR), starforming galaxies in MACS J1115+01 are offset to higher metallicity $(\sim 0.2$ dex) than the local Sloan Digital Sky Survey galaxies at a fixed mass range. In contrast, the MZR of RX J1532+30 is consistent with the local comparison sample. RX J1532+30 exhibits a bimodal radial metallicity distribution, with one branch showing a similar negative gradient to MACS J1115+01 $(-0.14 \pm 0.05 \mathrm{dex} / \mathrm{Mpc})$ and the other branch showing a positive radial gradient. The positive gradient branch in RX J1532+30 is likely caused by either interloper galaxies or an in-plane merger, indicating that cluster-scale abundance gradients probe cluster substructures and thus the dynamical state of a cluster. Most strikingly, we discover that neither the radial metallicity gradient nor the offset from the MZR is driven by the stellar mass. We compare our observations with Rhapsody-G cosmological hydrodynamical zoom-in simulations of relaxed galaxy clusters and find that the simulated galaxy cluster also exhibits a negative abundance gradient, albeit with a shallower slope $(-0.04 \pm 0.03 \mathrm{dex} / \mathrm{Mpc})$. Our observations suggest that the negative radial gradient originates from ram pressure stripping and/or strangulation processes in the cluster environments.
\end{abstract}

Key words: galaxies: clusters: individual: (MACS J1115+0129, RX J1532+3021) - Galaxy: abundances Galaxy: evolution

\section{INTRODUCTION}

Understanding the effect of cluster environment on galaxy formation and evolution is a central topic in extragalactic astronomy. A galaxy falling into the dense environment of a galaxy cluster experiences many high-speed encounters with the cluster members and tidal interactions with the dark matter halo of the cluster, leading to the removal of gas and disk destabilization (galaxy harassment; Moore et al. 1996). At the same time, the hot intracluster medium (ICM) interacts with the interstellar medium (ISM) of the galaxy and strips off its gas reservoir through ram pressure (ram pressure stripping; Gunn et al. 1972; Dressler \& Gunn 1983; Gavazzi et al. 1995). The galaxy loses a large fraction of the halo gas as it moves through the cluster, resulting in a gradual decline of star formation as it slowly runs out of fuel (strangulation; Larson et al. 1980). Additionally, if the dynamical friction time is sufficiently short, the galaxy will ultimately reach the cluster center and merge with the central brightest cluster galaxy (BCG, galactic cannibalism; Ostriker \& Tremaine 1975; Hausman \& Ostriker 1978; De Lucia \& Blaizot 2007). All of the above processes are directly associated with the mass and ICM gas distribution (e.g., Gunn et al. 1972; Dressler 1980; De Lucia \& Blaizot 2007; Bialas et al. 2015). The theoretical dark matter density profile of a cluster is universal (e.g., Navarro et al. 1997; Huss et al. 1999); however, the exact structure of the local mass and ICM gas density depends on the dynamical state of the cluster (Dressler \& Shectman 1988; Serna \& Gerbal 1996; Adami et al. 2005).
Gas-phase metallicity provides a fossil record of the formation history of galaxies, modulated by galactic-scale gas flows. Metallicity can trace the impact of mass and environment on galaxy evolution. The mass-metallicity relation (MZR) in the local universe has minimal dependence on environment, as shown by most observations and simulations of local field and cluster galaxies (Mouhcine et al. 2007; Cooper et al. 2008; Ellison et al. 2009; Davé et al. 2011; Pasquali et al. 2012; Scudder et al. 2012; Hughes et al. 2013). Galaxies in clusters tend to have slightly higher metallicity ( $\sim 0.04 \mathrm{dex})$ when compared to a control sample of field galaxies (Ellison et al. 2009). However, Ellison et al. (2009) find that the slight enhancement in metallicity is mainly driven by the presence of close companions, not simply the cluster membership. The metallicity of star-forming satellite galaxies at $z \sim 0$ shows a weak positive correlation with environmental overdensity; however, such a correlation is not seen in central galaxies (Peng \& Maiolino 2014). In high-density environments such as inner regions of the galaxy clusters at $z \sim 0$, the gas-phase metallicity is higher by $0.08-0.20$ dex than counterpart field galaxies (Mouhcine et al. 2007). Darvish et al. (2015) found that galaxies in the filamentary structure at $z \sim 0.5$ have $\sim 0.15$ dex higher metallicity than their counterpart field galaxies.

Studies at higher redshift $(z \sim 2)$ are inconclusive about the environmental dependence of metallicity (e.g., Kacprzak et al. 2015; Shimakawa et al. 2015; Tran et al. 2015; Valentino et al. 2015). Most studies on the environmental dependence of 
MZR compare galaxies residing in low- versus high-density environments (Mouhcine et al. 2007; Cooper et al. 2008; Scudder et al. 2012), but the local environment density in a cluster depends on the cluster-centric distance. Therefore, to assess the effect of the cluster environment on chemical evolution, it is essential to consider the cluster-centric distance.

Galaxy properties such as color, morphology, and luminosity show a strong dependence on the density (or cluster-centric distance) in galaxy clusters. The morphology-density relation shows that the elliptical galaxy fraction is higher in the cluster core than in the cluster outskirts (e.g., Dressler 1980; Houghton 2015). Similarly, the environmental dependence of the luminosity function shows that the most luminous (or most massive) galaxies exist in the densest parts of the universe, i.e., the cores of galaxy groups and clusters (Davis et al. 1988; Zandivarez \& Martínez 2011). In addition, the red galaxy fraction decreases with cluster-centric distance (Martínez et al. 2008; Ribeiro et al. 2013), implying an increasing star formation rate with cluster-centric distance. Simulations of galaxy clusters can reproduce the color distribution of cluster members by assuming a decreasing star formation rate after galaxies enter the cluster (Balogh et al. 2000). A recent study by Maier et al. (2016) finds a higher fraction of metal-rich galaxies in the accreted region of the galaxy cluster than galaxies in the infalling region. However, there is no comprehensive study on the radial dependence of cluster member metallicities.

The radial metallicity distribution can constrain the mass assembly of galaxy clusters and the effect of cluster environment on the evolution of individual galaxies within the cluster. Both simulations and X-ray observations find that the X-ray plasma in galaxy clusters has higher metallicities in the cluster core than at larger cluster radius (De Grandi \& Molendi 2001; HlavacekLarrondo et al. 2013), representing the excess average star formation in the cluster core. The observed negative radial gradients in ICM metallicity are often used as a constraint in galaxy cluster simulations (e.g., Kapferer et al. 2007; Cora et al. 2008; Martizzi et al. 2015; Rasia et al. 2015). Recent simulations by Martizzi et al. (2015) show that the ICM metallicities can be explained by a simple regulator model, driven by metal enrichment from stellar nucleosynthesis and gas accretion. Martizzi et al. (2015) and Rasia et al. (2015) also find a mild correlation between stellar metallicity and cluster-centric distance, with X-ray-luminous (cool-core) clusters exhibiting steeper metallicity gradients than non-cool-core clusters. These results imply that the chemical evolution of individual cluster galaxies is intimately linked with the local ICM properties of the cluster. Observations of the ISM gas-phase metallicity in galaxies as a function of the ICM density (or cluster-centric distance) provide new insights into the interplay between cluster galaxies and the cluster environment.

We present the first observations of the radial distribution of global ISM metallicity of star-forming galaxies in galaxy clusters. This work is based on optical spectra obtained with DEIMOS on the Keck II telescope. The large field of view of DEIMOS allows the observation of cluster members up to two times the virial radius $\left(\sim 2 \times r_{\text {vir }}\right)$ as compared to the small central region probed by X-ray observations $\left(\sim 200 \mathrm{kpc} \sim 0.2 \times r_{\text {vir }}\right)$. We investigate the global metallicity of star-forming galaxies as a function of radial distance in two X-ray-luminous clusters, MACS J1115 +0129 and $\mathrm{RX} \mathrm{J} 1532+3021$ at $z \sim 0.35$, and compare our observations with the Rhapsody-G simulations from Martizzi et al. (2015).
We organize this paper as follows. In Section 2, we describe the cluster sample selection, observations, data reduction, and derived quantities, including the metallicity diagnostics. The main results of this paper are presented in Section 3. In Section 4, we discuss the possible mechanisms responsible for the origin of a cluster-scale metallicity gradient. Throughout this work, we assume a standard $\Lambda$ CDM cosmology with $h=$ $0.696, \Omega_{\Lambda}=0.714$, and $\Omega_{M}=0.286$ (Hinshaw et al. 2013).

\section{OBSERVATIONS AND DATA REDUCTION}

\subsection{Cluster Sample Selection}

The data used in this paper are part of our Gravitationally Lensed-galaxies Observed With-Adaptive Optics (GLOW-AO) survey. For the GLOW-AO survey the galaxy clusters were selected from the Cluster Lensing And Supernova survey with Hubble (CLASH; Postman et al. 2012) clusters. Our GLOWAO survey was designed to detect lensed galaxies in the redshift range of $0.8<z<1.6$ that can be observed with adaptive optics. The CLASH galaxy clusters were originally selected from a sample of X-ray-bright clusters based on their gravitational lensing strength. The GLOW-AO survey is optimized for lensed galaxies to be followed up with AO observations using the OSIRIS instrument at the Keck I telescope. We therefore select clusters that have at least one bright star $\left(R_{\mathrm{AB}}<18.0\right)$ within a radius of $60^{\prime \prime}$ from the central BCG. In order to perform a comprehensive analysis of the effect of cluster environment on the abundance gradient, we choose galaxy clusters with at least 15 cluster members. This restricted our cluster sample to two out of the eight observed galaxy clusters, MACS J1115+0129 and RX J1532+3021.

MACS J1115+01 (Ebeling et al. 1998) and RX J1532+30 (Ebeling et al. 2010) are massive clusters with virial masses of $\sim 10^{14} M_{\odot}$ (Postman et al. 2012). Table 1 lists the derived physical and observational parameters for the selected clusters. X-ray morphology maps for both clusters exhibit minimal disturbances, highlighting the dynamically relaxed nature of the clusters (Allen et al. 2007). However, radio mini-halos were found around the central $200 \mathrm{kpc}$ of RX J1532+30, suggesting a possible dynamical disturbance in the central region of this cluster (Kale et al. 2013). No radio disturbance is found in MACS J1115+01. The nonuniform large-scale temperature distribution from X-ray observations of RX J1532+30 suggests a past minor merger with a cooler subcluster (Hlavacek-Larrondo et al. 2013). Spectroscopic data for MACS J1115+01 and RX $\mathrm{J} 1532+30$ are very limited. Our optical spectra provide important data to investigate dynamical structures of the clusters through the velocity and metallicity distributions of cluster members.

\subsection{Local Comparison Sample}

For our mass-metallicity study, we derive the local comparison sample from the Sloan Digital Sky Survey Data Release 7 (SDSS-DR7; York et al. 2000; Abazajian et al. 2009). Despite being at a lower redshift, SDSS galaxies were used as the comparison sample, as there is a little evolution in the ISM properties of galaxies between $z \sim 0.1$ and $z \sim 0.35$ (Kewley et al. 2013). We use publicly available MPA-JHU catalogs for the stellar masses and emission-line flux measurements of the SDSS-DR7 galaxies ${ }^{4}$ (Kauffmann

\footnotetext{
4 See http://wwwmpa.mpa-garching.mpg.de/SDSS/DR7/Data/ stellarmass.html.
} 
Table 1

X-Ray and Optically Derived Properties of Galaxy Cluster Sample

\begin{tabular}{|c|c|c|c|c|c|c|c|c|}
\hline Cluster & $z^{\mathrm{a}}$ & $\begin{array}{c}k T^{\mathrm{b}} \\
(\mathrm{keV})\end{array}$ & $\begin{array}{c}L_{\mathrm{Bol}}^{\mathrm{b}} \\
\left(10^{44} \mathrm{erg} \mathrm{s}^{-1}\right) \\
\end{array}$ & $\begin{array}{c}r_{\mathrm{vir}}^{\mathrm{c}} \\
(\mathrm{Mpc} / h) \\
\end{array}$ & $\begin{array}{c}M_{\mathrm{vir}} \mathrm{c} \\
\left(10^{15} M_{\odot} / h\right)\end{array}$ & $\bar{~} \bar{c}_{\mathrm{vir}}^{\mathrm{c}}$ & $\begin{array}{c}\sigma_{\mathrm{cl}}{ }^{\mathrm{d}} \\
\left(\mathrm{km} \mathrm{s}^{-1}\right)\end{array}$ & $\begin{array}{c}M_{\mathrm{vel}}^{\mathrm{d}} \\
\left(10^{15} M_{\odot} / h\right) \\
\end{array}$ \\
\hline MACS J1115+0129 & 0.352 & $8.0 \pm 0.4$ & $21.1 \pm 0.4$ & 1.78 & $1.13 \pm 0.10$ & $2.9 \pm 0.9$ & $960 \pm 147$ & $1.91 \pm 0.61$ \\
\hline RX J1532+3021 & 0.362 & $5.5 \pm 0.4$ & $20.5 \pm 0.9$ & 1.47 & $0.64 \pm 0.09$ & $3.8 \pm 1.7$ & $1487 \pm 213$ & $3.78 \pm 0.73$ \\
\hline
\end{tabular}

Notes.

${ }^{a}$ Redshift derived from SDSS spectra of central BCG.

${ }^{\mathrm{b}} k T$ is the temperature, and $L_{\mathrm{Bol}}$ is the total luminosity derived from the X-ray data (Postman et al. 2012).

${ }^{\mathrm{c}} r_{\mathrm{vir}}$ is the virial radius, $M_{\mathrm{vir}}$ is the total halo mass at the virial radius, and $c_{\mathrm{vir}}$ is the concentration parameter at the virial radius of the cluster derived by combining the strong- and weak-lensing observations (Merten et al. 2015).

d $\sigma_{\mathrm{cl}}$ is the radial velocity dispersion from our observations, and $M_{\mathrm{vel}}$ is the halo mass estimated from the velocity dispersion.

et al. 2003b; Brinchmann et al. 2004). We use a simple mass cut to match the stellar mass range of the SDSS sample to our DEIMOS cluster data. To ensure $>20 \%$ aperture coverage for each galaxy and to minimize the aperture effects (Kewley et al. 2005), we select SDSS galaxies within the redshift range of $0.05<z<0.10$. We select star-forming galaxies using the standard Baldwin-Phillips-Terlevich (BPT) diagram criteria described in Kewley et al. (2006). We further restrict the SDSS sample by selecting galaxies with signal-to-noise ratio $(\mathrm{S} / \mathrm{N}) \geqslant$ 5.0 for the following emission lines: [O II] $\lambda \lambda 3726,3729, \mathrm{H} \beta$ $\lambda 4861,[\mathrm{O}$ III] $\lambda \lambda 4959,5007$, [N II] $\lambda \lambda 6549,6583, \mathrm{H} \alpha \lambda 6563$, and [S II] $\lambda \lambda 6717,6731$. The size of the final sample to compare with our cluster data is 39,388 for MACS J1115+01 and 47,400 for RX J1532+30.

\subsection{Mask Design and Spectroscopy}

The mask for MACS J1115+01 was designed using publicly available Hubble Space Telescope (HST) images and photometric catalogs. Because the HST images cover only the central $\sim 120^{\prime \prime}$, we used Canada-France-Hawaii Telescope (CFHT) color images offered by C. J. Ma \& H. Ebeling (2016, private communication) to select potential cluster members and background lensed galaxies. Lensed galaxy candidates were identified based on their morphology, position around central BCG, and photometry. The highest-priority slits were placed on candidate lensed galaxies. The subsequent priorities of objects are listed in Table 2. Galaxies that can be observed with a natural guide star, i.e., an $R_{\mathrm{AB}}<18.0$ star at a separation of $<30^{\prime \prime}$ radius from the central BCG, are referred to as NGS. Similarly, galaxies referred to as LGS can be observed with a laser guide star, i.e., they have a star with $R_{\mathrm{AB}}<18.0$ within a $60^{\prime \prime}$ radius. The remainder of the mask was filled with galaxies with $R_{\mathrm{AB}}<24$. We designed each mask to have at least two stars with $R_{\mathrm{AB}}<15$ on each side to ensure proper mask alignment. Our GLOW-AO survey targeted primarily strongly lensed galaxies. The strong-lensing region ( central $500 \mathrm{kpc}$ ) of the cluster mask was filled with lensed galaxy candidates, thus deliberately missing cluster members in the central part of the cluster.

\subsection{DEIMOS Observations}

All observations were conducted with DEIMOS (Faber et al. 2003, pp. 1657-69) at the KECK II telescope on UTC 2014 February 24. We used the 600ZD grating at the central wavelength of $7500 \AA$ and the OG550 filter. Our spectra had a wavelength coverage of 5500-9800 $\AA$ with a dispersion of $0.65 \AA$ pixel $^{-1}$ and spectral resolution of $R \sim 2000$. The slit
Table 2

Priority List of Targets

\begin{tabular}{lc}
\hline \hline Objects & Priorities $^{\mathrm{a}}$ \\
\hline Lensed candidates & 1000 \\
Photometric $z(0.8<z<2.5)$ & 900 \\
NGS galaxies & 800 \\
LGS galaxies & 600 \\
Galaxies $\left(R_{\mathrm{AB}}<24.0\right)$ & 100 \\
\hline
\end{tabular}

Note. "Objects" represents the category of galaxies on the DEIMOS mask.

a "Priorities" is the number assigned by us during the DEIMOS mask designing.

width for all observations was $1^{\prime \prime}$, and all slits had a constant position angle the same as the DEIMOS mask. A DA-type white dwarf star, G191-B2B (Rauch et al. 2013), with $V_{\mathrm{AB}}=11.74$ was used for flux calibration. We developed a robust technique to flux-calibrate the DEIMOS observations by deriving the separate sensitivity function of individual CCDs of the spectrograph (Gupta et al. 2016). The absolute fluxcalibrated spectrum of the star G191-B2B was obtained from the ESO archive. ${ }^{5}$ Table 3 summarizes the observations for the data used in this paper. The mask for MACS J1115+01 was observed with a total integration time of $1 \mathrm{hr}$ split into $3 \times 20$ minute exposures to eliminate cosmic rays. The mask for RX $\mathrm{J} 1532+30$ had a total integration time of $1 \mathrm{hr} 40$ minutes $(5 \times 20$-minute exposures $)$. Each mask had $\approx 130$ slits, and the average seeing during observations was $\sim 0$ ". 8 .

\subsection{Data Reduction}

We reduced the data in two stages. First, we used the publicly available spec2d pipeline written by Michael Cooper (Cooper et al. 2011) to reduce the raw data. The pipeline generates 2D slit spectra after performing the bias removal, flatfielding, slit-tilt correction, cosmic-ray rejection, and wavelength calibration. Second, we used our IDL-based code (Gupta et al. 2016) to correct for atmospheric absorption, derive instrument sensitivity, and flux-calibrate the 2D slit spectra.

For telluric correction, we used the long-slit mode observation of the standard star G191-B2B, which is a DA-type white dwarf star with an effective temperature of 60,920 K (Rauch et al. 2013). The atmospheric absorption profile convolved with an instrument response curve was derived from the stellar 1D spectrum after removing the intrinsic stellar absorption features and the underlying blackbody profile. All 2D spectra were

5 See http://www.eso.org/sci/observing/tools/standards/spectra.html. 
Table 3

Observation Summery

\begin{tabular}{|c|c|c|c|c|c|}
\hline Targets & $\alpha(\mathrm{J} 2000)^{\mathrm{a}}$ & $\bar{c} \delta(\mathrm{J} 2000)^{\mathrm{a}}$ & Mask & Airmass & Exposure Time \\
\hline MACS J1115+01 & $11: 15: 52.1^{\mathrm{a}}$ & $+01: 29: 53^{\mathrm{a}}$ & Multi-slit & 1.06 & $3 \times 1200 \mathrm{~s}$ \\
\hline RX J1532+30 & $15: 32: 53.8^{\mathrm{a}}$ & $+30: 20: 58^{\mathrm{a}}$ & Multi-slit & 1.09 & $5 \times 1200 \mathrm{~s}$ \\
\hline G191-B2B ${ }^{b}$ & $05: 05: 30.6$ & $+52: 49: 54$ & Long-slit & 1.19 & $45 \mathrm{~s}$ \\
\hline G191-B2B ${ }^{c}$ & $05: 05: 30.6$ & $+52: 49: 54$ & No mask (CCD1) & 1.19 & $15 \mathrm{~s}$ \\
\hline G191-B2B ${ }^{c}$ & $05: 05: 30.6$ & $+52: 49: 54$ & No mask (CCD2) & 1.19 & $15 \mathrm{~s}$ \\
\hline G191-B2B ${ }^{c}$ & $05: 05: 30.6$ & $+52: 49: 54$ & No mask (CCD3) & 1.19 & $15 \mathrm{~s}$ \\
\hline G191-B2B ${ }^{c}$ & $05: 05: 30.6$ & $+52: 49: 54$ & No mask (CCD4) & 1.19 & $15 \mathrm{~s}$ \\
\hline
\end{tabular}

Notes. Seeing $\sim 0{ }^{\prime \prime} 8$.

${ }^{a}$ Coordinates of the BCG centroid (J2000).

${ }^{\mathrm{b}}$ Used for telluric correction and long-slit mode flux calibration.

c Slitless mode flux calibration.
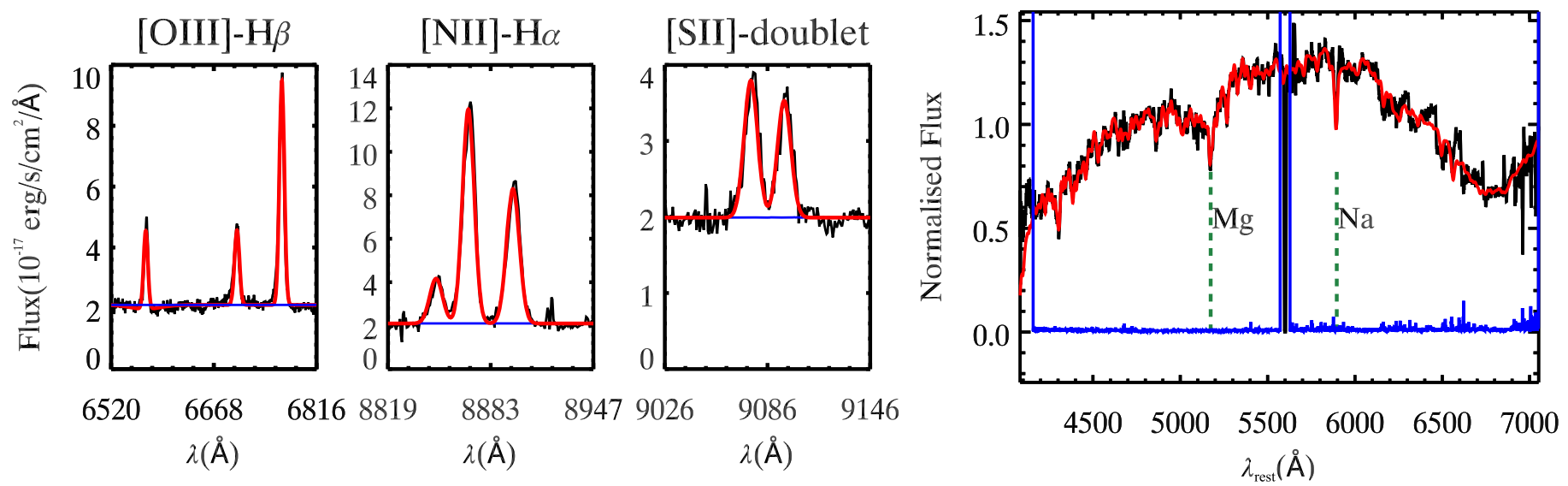

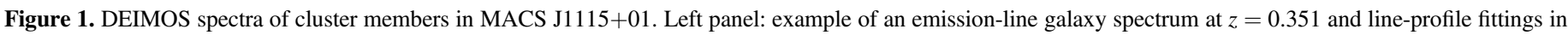

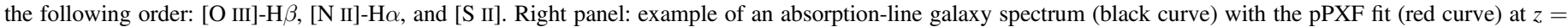
0.347 . The blue curve shows the error spectrum supplied to the pPXF.

divided by the derived sensitivity curve to correct for instrument sensitivity and telluric absorption.

We used the process described in Gupta et al. (2016) to fluxcalibrate 2D slit spectra from DEIMOS. The 1D spectrum of the observed objects was extracted by fitting a Gaussian curve to the spatial slit profile and integrating the flux within the FWHMs. For galaxies where Gaussian fitting was not possible, we used FWHM calculated from the point-spread function of alignment stars on the DEIMOS mask to extract 1D spectrum.

\subsection{Emission-line Fitting}

We visually inspected all 2D slit spectra to estimate an initial redshift by identifying strong emission lines such as [O II] $\lambda \lambda 3726,3729, \mathrm{H} \beta,[\mathrm{O}$ III] $\lambda 4959,5007, \mathrm{H} \alpha$, and $[\mathrm{N}$ II $] \lambda 6583$. To determine the need to correct for stellar absorption before extracting emission-line fluxes, we select emission-line-dominated galaxies that have sufficient $\mathrm{S} / \mathrm{N}$ in the continuum to fit stellar absorption spectra (20\% of the emission-line sample). Continuum subtraction was performed with a penalized pixelfitting routine (pPXF; Cappellari \& Emsellem 2004) based on the MILES stellar population models (Vazdekis et al. 2010). We find that the difference in line ratios before and after continuum subtraction is largest in $\log ([\mathrm{O} \mathrm{IIII}] / \mathrm{H} \beta)(\sim 0.05 \mathrm{dex})$, whereas for other line ratios the difference is negligible. The difference in N2S2-diagnostic metallicity (See Section 2.10) before and after continuum subtraction is less than $0.04 \mathrm{dex}$, smaller than the statistical error of our observations
( $~ 0.07$ dex $)$. Therefore, stellar absorption plays a negligible role in the emission-line sample tested above. A majority $(\sim 80 \%)$ of the members of our emission-line sample do not have sufficient $\mathrm{S} / \mathrm{N}$ in the continuum. We do not perform stellar absorption fitting before extracting emission lines for consistent analysis across the entire sample.

Multiple Gaussian profiles were fit to emission lines simultaneously depending on their separation in wavelength. For the [O II] doublet $\lambda \lambda 3726,3729$, we fit a double Gaussian with five free parameters: redshift, flux peak-1, flux peak-2, line width, and continuum level. A similar double-Gaussian function was used for the [S II] $\lambda \lambda 6717,6731$ doublet. We fit a triple Gaussian to the [N II], $\mathrm{H} \alpha$ emission lines using six free parameters: redshift, flux-H $\alpha$, flux-[N II], line width-H $\alpha$, line width-[N II], and continuum level. The ratio of [N II] $\lambda 6548$ to [N II] $\lambda 6583$ was constrained to its theoretical value of $1 / 3$ (Osterbrock 1989). The [O III], $\mathrm{H} \beta$ emission line group was simultaneously fit using four Gaussians with eight free parameters: redshift, flux-[O III], width-[O III], flux-H $\beta$, width- $\mathrm{H} \beta$, flux absorption- $\mathrm{H} \beta$, width absorption- $\mathrm{H} \beta$, and continuum level. The left panel of Figure 1 presents the example of emission-line fits for cluster members in MACS $\mathrm{J} 1115+01$ at $z=0.351$. We identified 73 redshifts out of 127 slits in the redshift range of $0.05<z<1.13$ for the mask MACS J1115+01. For RX J1532+30, 83 redshifts were identified out of the 139 slits in the redshift range of $0.036<z<0.992$. Ratios of major emission lines, [O III]/ 
Table 4

Data Table-Emission-line Galaxies for MACS J1115+01

\begin{tabular}{|c|c|c|c|c|c|c|c|}
\hline ID & $z^{\mathrm{a}}$ & $r_{\text {pro }}{ }^{\mathrm{b}}$ & $\overline{l o g}\left(M_{*} / M_{\odot}\right)^{\mathrm{c}}$ & $\log ([\mathrm{O} \text { III }] / H \beta)^{d}$ & $\log ([\mathrm{N} \mathrm{II}] / \mathrm{H} \alpha)^{\mathrm{d}}$ & $\overline{l o g}([\mathrm{~S} \text { II }] / \mathrm{H} \alpha)^{\mathrm{d}}$ & $\overline{\log ([\mathrm{N} \mathrm{II}] /[\mathrm{S} \mathrm{II}])^{\mathrm{d}}}$ \\
\hline $1115020-0$ & 0.353 & 528.79 & 10.0 & $\ldots \pm$ & $-0.39 \pm 0.05$ & $-0.63 \pm 0.04$ & $0.23 \pm 0.06$ \\
\hline $1115026-0$ & 0.351 & 1081.7 & 10.6 & $0.53 \pm 0.02$ & $-0.17 \pm 0.01$ & $-0.44 \pm 0.01$ & $0.28 \pm 0.01$ \\
\hline $1115042-0$ & 0.341 & 785.53 & 9.53 & $-0.14 \pm 0.03$ & $-0.46 \pm 0.03$ & $-0.54 \pm 0.04$ & $0.088 \pm 0.05$ \\
\hline $1115069-0$ & 0.352 & 875.36 & 10.9 & $-0.30 \pm 0.08$ & $-0.63 \pm 0.08$ & $-0.12 \pm 0.02$ & $-0.51 \pm 0.08$ \\
\hline $1115072-0$ & 0.349 & 920.02 & 9.57 & $\ldots \pm \ldots$ & $-0.67 \pm 0.09$ & $-0.52 \pm 0.04$ & $-0.15 \pm 0.09$ \\
\hline $1115077-0$ & 0.354 & 2340.0 & 9.37 & $-0.11 \pm 0.02$ & $-0.65 \pm 0.1$ & $-0.39 \pm 0.02$ & $-0.26 \pm 0.1$ \\
\hline 1115088-0 & 0.350 & 1379.6 & 9.51 & $\ldots \pm \ldots$ & $\ldots \pm \ldots$ & $-0.035 \pm 0.2$ & $\ldots \pm$ \\
\hline 1115096-0 & 0.345 & 1077.0 & 9.27 & $-0.60 \pm 0.1$ & $-0.32 \pm 0.07$ & $-0.34 \pm 0.05$ & $0.022 \pm 0.08$ \\
\hline $1115103-0$ & 0.355 & 743.73 & 10.2 & $\ldots \pm \ldots$ & $-0.28 \pm 0.07$ & $-0.59 \pm 0.07$ & $0.31 \pm 0.07$ \\
\hline $1115112-0$ & 0.360 & 854.36 & 9.66 & $\ldots \pm \ldots$ & $-0.29 \pm 0.05$ & $-0.28 \pm 0.06$ & $-0.0068 \pm 0.07$ \\
\hline $1115116-0$ & 0.346 & 688.04 & 9.60 & $-0.45 \pm 0.1$ & $-0.29 \pm 0.04$ & $-0.38 \pm 0.04$ & $0.091 \pm 0.05$ \\
\hline $1115117-0$ & 0.350 & 916.24 & 10.3 & $-0.62 \pm 0.05$ & $-0.47 \pm 0.02$ & $-0.65 \pm 0.02$ & $0.18 \pm 0.03$ \\
\hline $1115119-0$ & 0.357 & 2487.8 & 10.1 & $-0.56 \pm 0.05$ & $-0.47 \pm 0.04$ & $-0.55 \pm 0.02$ & $0.076 \pm 0.04$ \\
\hline $1115121-0$ & 0.345 & 853.26 & 9.70 & $\ldots \pm \ldots$ & $-0.28 \pm 0.09$ & $-0.31 \pm 0.07$ & $0.021 \pm 0.1$ \\
\hline
\end{tabular}

Notes.

${ }^{\mathrm{a}} z$ represents the best-fitting redshift to the DEIMOS spectra using the emission lines.

${ }^{\mathrm{b}} r_{\text {pro }}$ is the projected cluster-centric distance of the cluster members from the central BCG.

${ }^{\mathrm{c}} \log \left(M_{*} / M_{\odot}\right)$ is the stellar mass derived from the SDSS photometry (See Section 2.9).

${ }^{\mathrm{d}} \log ([\mathrm{O} \mathrm{III]} / \mathrm{H} \beta), \log ([\mathrm{N} \mathrm{II}] / \mathrm{H} \alpha), \log ([\mathrm{S} \mathrm{II}] / \mathrm{H} \alpha)$, and $\log ([\mathrm{N} \mathrm{II}] /[\mathrm{S}$ II $])$ are the emission-line ratios derived from the DEIMOS spectra (see Section 2.6).

Table 5

Data Table-Emission-line Galaxies for RX J1532+30

\begin{tabular}{|c|c|c|c|c|c|c|c|}
\hline ID & $z^{\mathrm{a}}$ & $r_{\text {pro }}{ }^{a}$ & $\log \left(M_{*} / M_{\odot}\right)^{\mathrm{a}}$ & $\log ([\mathrm{O} \mathrm{III}] / \mathrm{H} \beta)^{\mathrm{a}}$ & $\log ([\mathrm{N} \text { II }] / \mathrm{H} \alpha)^{\mathrm{a}}$ & $\log ([\mathrm{S} \mathrm{II}] / \mathrm{H} \alpha)^{\mathrm{a}}$ & $\log ([\mathrm{N} \mathrm{II}] /[\mathrm{S} \mathrm{II}])^{\mathrm{a}}$ \\
\hline $1532008-1$ & 0.363 & $\ldots$ & 10.5 & $\ldots \pm$ & $-0.23 \pm 0.04$ & $\ldots \pm \ldots$ & $\ldots \pm \ldots$ \\
\hline $1532017-0$ & 0.353 & 772.08 & 9.95 & $0.44 \pm 0.05$ & $-0.99 \pm 0.1$ & $-0.55 \pm 0.03$ & $-0.43 \pm 0.1$ \\
\hline $1532023-0$ & 0.357 & 979.84 & 10.1 & $\ldots \pm$ & $-0.22 \pm 0.1$ & $-0.33 \pm 0.08$ & $0.11 \pm 0.1$ \\
\hline $1532032-0$ & 0.357 & 1158.7 & 9.03 & $\ldots \pm \ldots$ & $-0.72 \pm 0.1$ & $-0.40 \pm 0.03$ & $-0.32 \pm 0.1$ \\
\hline $1532034-0$ & 0.357 & 1301.9 & 8.60 & $0.47 \pm 0.03$ & $-0.77 \pm 0.09$ & $-0.51 \pm 0.05$ & $-0.26 \pm 0.1$ \\
\hline $1532066-0$ & 0.364 & 351.44 & 10.2 & $\ldots \pm \ldots$ & $\ldots \pm \ldots$ & $\ldots \pm \ldots$ & $\ldots \pm \ldots$ \\
\hline $1532086-0$ & 0.354 & 619.21 & 10.3 & $0.14 \pm 0.08$ & $-0.32 \pm 0.02$ & $-0.40 \pm 0.02$ & $0.086 \pm 0.03$ \\
\hline $1532087-0$ & 0.354 & 636.69 & 9.13 & $0.40 \pm 0.02$ & $-1.3 \pm 0.1$ & $-0.62 \pm 0.01$ & $-0.64 \pm 0.1$ \\
\hline $1532093-0$ & 0.354 & 934.69 & 10.2 & $0.15 \pm 0.01$ & $-0.38 \pm 0.02$ & $-0.43 \pm 0.01$ & $0.053 \pm 0.02$ \\
\hline $1532098-0$ & 0.361 & 1238.5 & 9.84 & $-0.43 \pm 0.03$ & $-0.37 \pm 0.02$ & $-0.58 \pm 0.009$ & $0.21 \pm 0.02$ \\
\hline $1532102-0$ & 0.355 & 1491.3 & 10.0 & $-0.76 \pm 0.04$ & $-0.44 \pm 0.02$ & $-0.57 \pm 0.009$ & $0.14 \pm 0.02$ \\
\hline $1532110-0$ & 0.340 & 1685.1 & 9.27 & $-0.12 \pm 0.1$ & $-0.31 \pm 0.07$ & $-0.10 \pm 0.06$ & $-0.21 \pm 0.08$ \\
\hline $1532125-0$ & 0.343 & 2200.3 & 10.1 & $-0.56 \pm 0.05$ & $\ldots \pm \ldots$ & $\ldots \pm \ldots$ & $\ldots \pm \ldots$ \\
\hline
\end{tabular}

Note.

a Same as Table 4, but for galaxy cluster RX J1532+30.

$\mathrm{H} \beta,[\mathrm{N} \mathrm{II}] / \mathrm{H} \alpha,[\mathrm{S} \mathrm{II}] / \mathrm{H} \alpha$, and $[\mathrm{N} \mathrm{II}] /[\mathrm{S} \mathrm{II}]$, for the cluster members in MACS $\mathrm{J} 1115+01$ and RX $\mathrm{J} 1532+30$ are given in Tables 4 and 5 , respectively.

\subsection{Absorption-line Galaxies}

Spectra with no apparent emission lines but with a strong continuum were visually inspected for $\mathrm{Na} \lambda 5872, \mathrm{Mg} \lambda 5174$, and Balmer absorption lines, to estimate an initial redshift. We performed single stellar population fitting using the pPXF to fine-tune the redshifts of the absorption-line galaxies. We used the MILES stellar population models (Vazdekis et al. 2010) to best match the spectral resolution of our observations. The skylines and bad pixels were masked before fitting the continuum models. The right panel in Figure 1 shows the example of a fit from pPXF for a cluster member in MACS $\mathrm{J} 1115+01$ at $z=0.347$. Using pPXF, we calculated the redshift of eight absorption-line galaxies for MACS J1115+01 (Table 6) and 15 galaxies for RX J1532+30 (Table 7).

\subsection{Active Galatic Nucleus (AGN) Removal}

Abundance calibrations using strong emission lines are only robust for star-forming galaxies. Therefore, we need to remove AGNs from our cluster member sample. Galaxies can be classified on the basis of their global gas excitation properties 
Table 6

Data Table-Absorption-line Galaxies for MACS J1115+01

\begin{tabular}{lccc}
\hline \hline ID & $z^{\mathrm{a}}$ & $r_{\text {pro }}{ }^{\mathrm{b}}$ & $\log \left(M_{*} / M_{\odot}\right)^{\mathrm{c}}$ \\
\hline 1115025 & 0.347 & 728.38 & 10.7 \\
1115043 & 0.354 & 1659.6 & 10.5 \\
1115057 & 0.359 & 399.22 & 10.6 \\
1115064 & 0.351 & 613.48 & 10.9 \\
1115076 & 0.351 & 1111.1 & 10.5 \\
1115098 & 0.353 & 876.71 & 10.3 \\
1115100 & 0.352 & 1998.4 & 11.1 \\
1115115 & 0.351 & 752.85 & 11.4 \\
\hline
\end{tabular}

Notes.

${ }^{a} z$ represents the best-fitting redshift to the DEIMOS spectra using the absorption lines (see Section 2.7).

${ }^{\mathrm{b}} r_{\text {pro }}$ is the projected cluster-centric distance of the cluster members from the central BCG.

${ }^{\mathrm{c}} \log \left(M_{*} / M_{\odot}\right)$ is the stellar mass derived from the SDSS photometry (see Section 2.9).

on the BPT diagram (Baldwin et al. 1981; Phillips et al. 1986; Veilleux \& Osterbrock 1987). The hard ionizing radiation field from an AGN increases the intensity of the metal ion forbidden lines (e.g., $\mathrm{N}^{+}, \mathrm{O}^{++}$) as compared to the hydrogen recombination lines. The presence of large-scale shocks or an aged stellar population in post-starburst galaxies also results in enhanced intensity of emission lines from the metal ions. Therefore, galaxies with the presence of AGNs/evolved hot stars and/or shocks form a separate group on the BPT diagram than the starforming galaxies (Kewley et al. 2006; Sarzi et al. 2010; Rich et al. 2011; Yan \& Blanton 2012; Alatalo et al. 2016). Kewley et al. (2011, hereafter Ke01) developed a theoretical "maximum starburst line" on the BPT diagram using a combination of stellar population synthesis, photoionization, and shock models. An "empirical star-forming line" was developed by Kauffmann et al. (2003b, hereafter Kau03) using the SDSS data. $\mathrm{Ke} 01$ (red curve) and Kau03 (blue curve) classify galaxies into AGN, composite, and purely star forming, based on their location on the BPT diagram (Figure 2). The AGN versus starforming classification was developed for $z \sim 0$ galaxies. Because the redshift evolution between $z \sim 0$ and $z \sim 0.35$ is negligible on the BPT diagrams, the $z \sim 0$ classification can be used for our cluster sample at $z \sim 0.35$. Our results remain unchanged even if we use the new redshift-dependent classification by Kewley et al. (2013). For analysis of metallicity gradients, we use galaxies below the $\mathrm{Ke} 01$ line, thus incorporating both the star-forming and the composite galaxies.

\subsection{Stellar Mass}

We derived the stellar mass from SDSS broadband photometry $(u, g, r, i, z)$ using the Le Phare package (Arnouts et al. 1999; Ilbert et al. 2006) with the stellar templates from Bruzual \& Charlot (2003) and a Chabrier initial mass funcation (IMF; Chabrier 2003). The stellar templates have seven exponentially decreasing star formation models $\left(\mathrm{SFR} \propto e^{-t / \tau}\right)$ with $\tau=0.1,0.3,1,2,3,5,10,15$, and $30 \mathrm{Gyr}$ and three metallicities. To correct for dust attenuation in stellar continuum, the Calzetti et al. (2000) models were used with $E(B-V)=0-0.6$. The stellar templates with stellar population ages from 0 to $13 \mathrm{Gyr}$ were used. Throughout this paper, we have used the median of the generated mass
Table 7

Data Table-Absorption-line Galaxies for MACS J1532+30

\begin{tabular}{lccc}
\hline \hline ID & $z^{\mathrm{a}}$ & $r_{\text {pro }}{ }^{\mathrm{a}}$ & $\log \left(M_{*} / M_{\odot}\right)^{\mathrm{a}}$ \\
\hline 1532009 & 0.359 & 63.504 & 10.7 \\
1532012 & 0.359 & 338.83 & 10.5 \\
1532018 & 0.361 & 904.93 & 10.7 \\
1532019 & 0.364 & 915.37 & 10.8 \\
1532027 & 0.360 & 1090.4 & 10.8 \\
1532031 & 0.359 & 1171.7 & 10.9 \\
1532065 & 0.358 & 382.43 & 10.6 \\
1532069 & 0.356 & 460.26 & 10.5 \\
1532071 & 0.359 & 500.72 & 10.4 \\
1532075 & 0.361 & 506.15 & 10.3 \\
1532077 & 0.364 & 508.77 & 9.66 \\
1532088 & 0.362 & 734.37 & 10.2 \\
1532090 & 0.360 & 805.38 & 10.6 \\
1532100 & 0.362 & 1265.8 & 10.4 \\
1532112 & 0.360 & 1740.8 & 11.1 \\
\hline
\end{tabular}

Note.

${ }^{\mathrm{a}}$ Same as Table 6, but for galaxy cluster RX J1532+30.

distribution as the stellar mass of our galaxies. The typical error from Le Phare in the mass estimation from the SDSS photometry is 0.2 dex.

\subsection{ISM Parameters}

We use a new theoretical calibration by Dopita et al. (2016) to derive the global metallicities for our selected cluster member galaxies. Direct measurement of the ISM metallicity requires observations of extremely weak auroral emission lines such as [O III] $\lambda 4363$, which is difficult for high-redshift galaxies. Thus, the metallicity is often determined via theoretical and empirical calibrations based on strong emission lines such as $\mathrm{H} \alpha$ and [N $\mathrm{NI}$. The strength of these emission lines depends on the ionization conditions in the galaxies, i.e., ionization parameter and ISM pressure. The new calibration by Dopita et al. (2016) using the $\log ([\mathrm{N} \mathrm{II}] / \mathrm{H} \alpha)$ and $\log ([\mathrm{N} \mathrm{II}] /[\mathrm{S} \mathrm{II}])$ ratios is independent of ionization conditions and scales linearly with the oxygen abundance $(12+\log (\mathrm{O} / \mathrm{H}))$, a proxy for the total metal content.

We use the Dopita et al. (2016) calibration, given by

$$
\begin{aligned}
& 12+\log (\mathrm{O} / \mathrm{H})=8.77+\log ([\mathrm{N} \mathrm{II}] /[\mathrm{S} \mathrm{II}]) \\
& +0.264 \log ([\mathrm{N} \mathrm{II}] / \mathrm{H} \alpha) \text {, }
\end{aligned}
$$

to derive the metallicity for both our cluster galaxies and the local comparison sample. We require an $\mathrm{S} / \mathrm{N}$ of 3 for all necessary emission lines to derive the metallicity. Using an independent metallicity calibration such as the one from Pettini \& Pagel (2004, N2) does not affect our results. We also determine the ionization properties of the cluster galaxies using $[\mathrm{O}$ III] $] / \mathrm{H} \beta$ and $[\mathrm{N} \mathrm{II]} / \mathrm{H} \alpha$. We found no statistically significant relationship between the ionization state of the gas and cluster radius, given our small sample size.

\section{RESULTS}

\subsection{Cluster Member Identification}

We identified cluster members based on the radial velocity of galaxies around the redshift of the central BCG. The peak of the redshift distribution for each mask coincides with the 

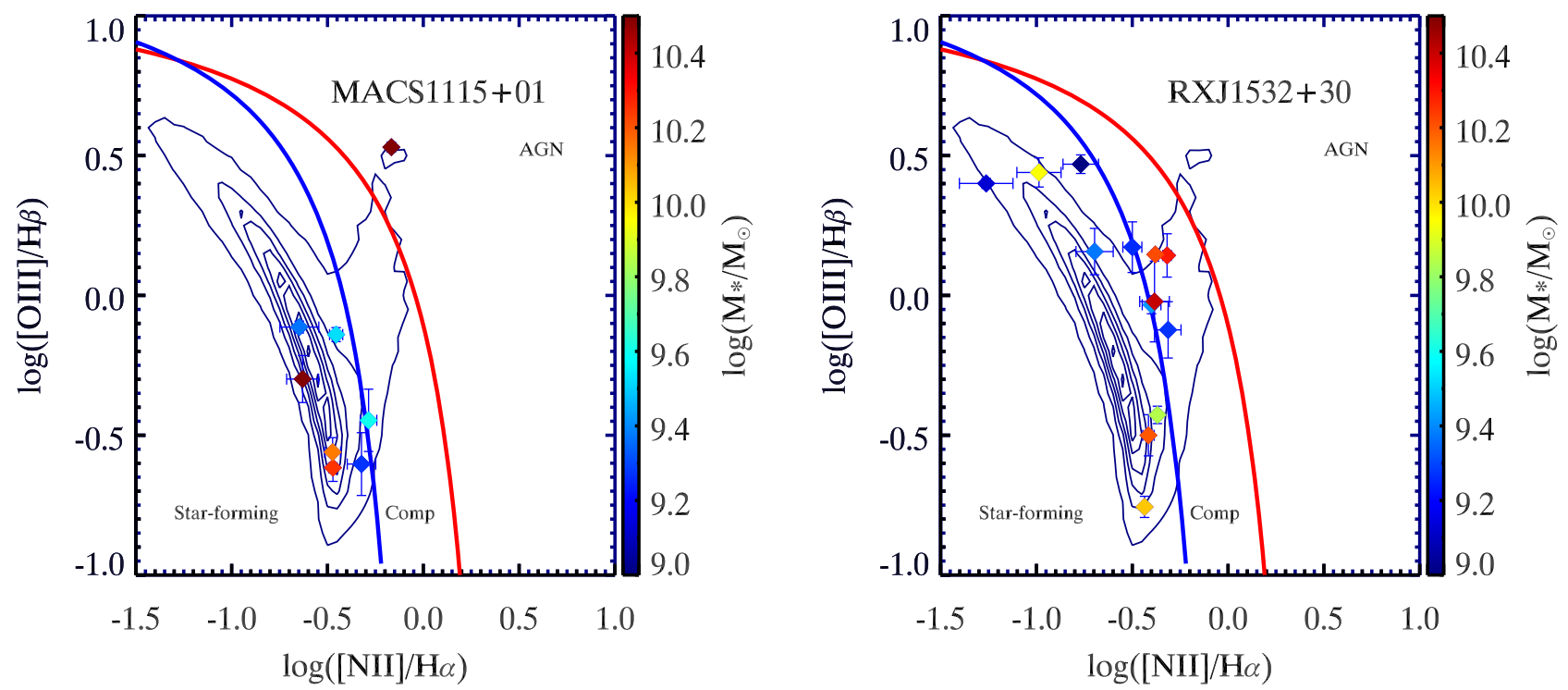

Figure 2. Optical BPT diagnostic diagrams for the observed cluster members for MACS J1115+01 (left panel) and RX J1532+30 (right panel). The data points in each panel are color-coded with stellar mass $\left(\log \left(M_{*} / M_{\odot}\right)\right)$. The red (Kewley et al. 2001) and blue (Kauffmann et al. 2003a) curves divide the galaxies into three subclasses, i.e., star-forming, composite, and AGN. The blue contours show line ratios for the local comparison sample. AGN galaxies are removed to ensure the starforming sample for the optical diagnostics.
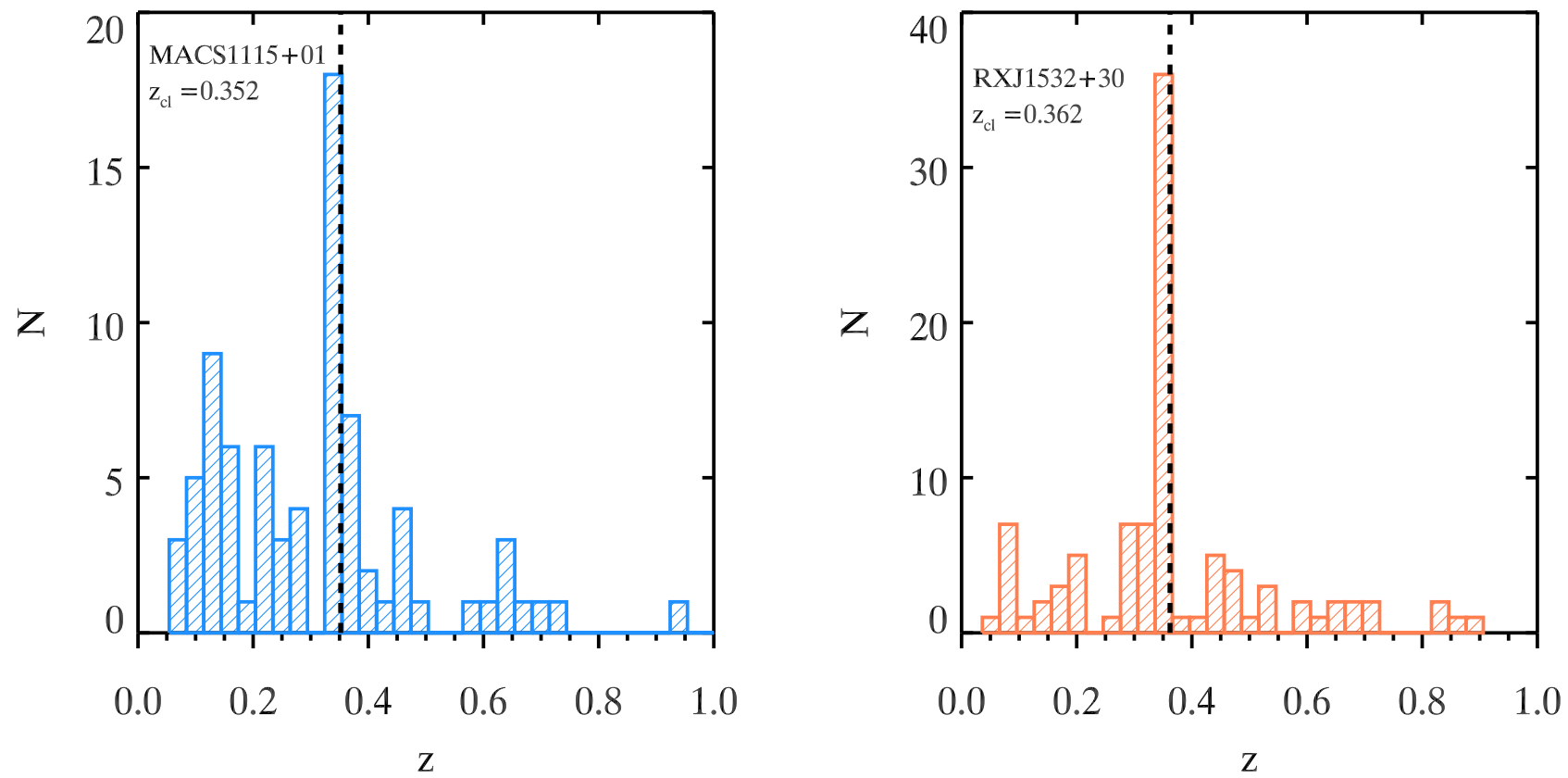

Figure 3. Redshift distribution of all objects observed on the DEIMOS mask. Left panel: MACS J1115+01; right panel: RX J1532+30. The black line in each panel indicates the redshift of the clusters. The peak in the histogram at the cluster redshifts clearly indicates detection of cluster members.

redshift of the central BCG of the respective clusters (Figure 3). We used an initial radial velocity cut of $\pm 10,000 \mathrm{~km} \mathrm{~s}^{-1}$ and a further $2.5 \sigma$ clip on the radial velocity with a basic outlier rejection.

We identified 22 cluster members for MACS J1115+01, of which 14 are emission-line galaxies. The radial velocity distribution of selected cluster members is represented by the blue bars in Figure 4 . The normality in velocity dispersion has been used to characterize the dynamically relaxed state of a galaxy cluster (e.g., Yahil \& Vidal 1977; Ribeiro et al. 2013). A one-sided Kolmogorov-Smirnov (K-S) test yields a probability of 55\% for the velocity distribution of MACS J1115+01 to be drawn from the normal distribution, indicating the dynamically relaxed state of the galaxy clusters. The velocity dispersion for MACS J1115+01 is $\sigma_{\mathrm{cl}}=960 \pm 147 \mathrm{~km} \mathrm{~s}^{-1}$. Assuming virialization, the total mass of MACS J1115+01 is $M_{\text {vel }}=(1.91 \pm 0.61) \times 10^{15} M_{\odot} / h$, which is within the $1 \sigma$ error of the virial mass calculated from the gravitational lensing observations $\left(M_{\mathrm{vir}}=1.13 \times 10^{15} M_{\odot} / h\right.$; Merten et al. 2015, Table 1). We find no obvious dependence of the stellar mass of cluster member galaxies on the position around the cluster center, for both emission-line (Figure 5, left panel, diamonds) and absorption-line (Figure 5, left panel, circles) systems.

For RX $\mathrm{J} 1532+30$, we identify 36 cluster members, of which 21 are emission-line galaxies. The cluster member mass of RX J1532+30 also appears to be independent of the spatial 


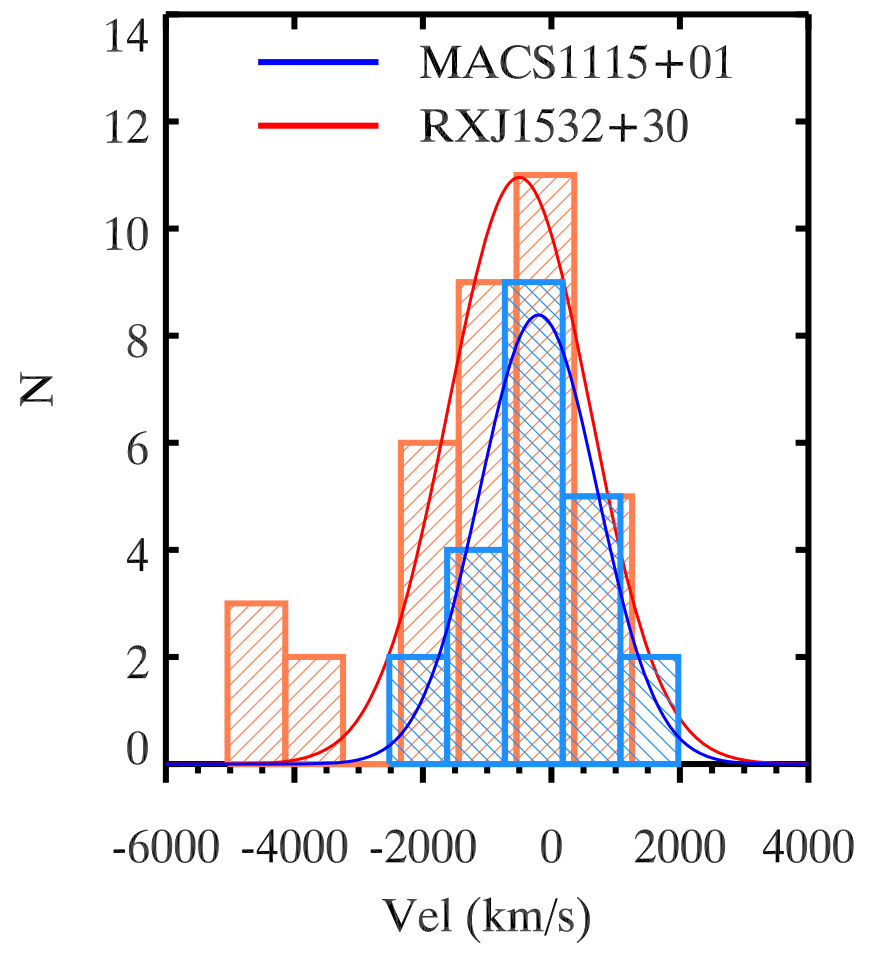

Figure 4. Radial velocity distribution of selected cluster members. The cluster members for MACS $\mathrm{J} 1115+01$ are shown in blue and those for RX J1532+30 in red. Solid lines indicate the Gaussian profiles of the velocity distribution, clearly showing the different dynamical states of the two clusters.

position around the central BCG (Figure 5, right panel). For $\mathrm{RX} \mathrm{J1532+30,} \mathrm{the} \mathrm{probability} \mathrm{of} \mathrm{the} \mathrm{radial} \mathrm{velocity} \mathrm{distribu-}$ tion (Figure 4, red bars) to be drawn from a normal distribution is only $5 \%$ according to the $\mathrm{K}-\mathrm{S}$ test. The observed velocity dispersion for RX $\mathrm{J} 1532+30$ is $\sigma_{\mathrm{cl}}=1487 \pm 213 \mathrm{~km} \mathrm{~s}^{-1}$. Assuming a virialized state for the cluster RX J1532+30, the total cluster mass estimated from the velocity dispersion would be $M_{\mathrm{vel}}=(3.78 \pm 0.73) \times 10^{15} M_{\odot} / h$, nearly six times the mass calculated from the gravitational lensing observations $\left(M_{\text {vir }}=0.64 \times 10^{15} M_{\odot} / h\right.$; Merten et al. 2015 , Table 1$)$. We discuss the discrepancy in the mass estimation from the radial velocity dispersion and gravitational lensing in Section 4.1.

\subsection{Mass-Metallicity Relation}

In Figure 6, we show the MZR for the star-forming cluster members in the two clusters (colored diamonds) and compare the cluster MZR with the MZR for the local sample (contours). The metallicities of cluster members and the SDSS sample were derived using the strong emission line diagnostics $\log ([\mathrm{N} \mathrm{II}] / \mathrm{H} \alpha)$ and $\log ([\mathrm{N} \mathrm{II}] /[\mathrm{S} \mathrm{II}])$ (See Section 2.10). The stellar mass was derived from SDSS photometry (Section 2.9). After applying an $\mathrm{S} / \mathrm{N}$ cut of 3 for the necessary emission lines, the metallicities could be calculated for 12 and 16 cluster members for MACS J1115+01 and RX J1532+30, respectively.

Both clusters show a positive correlation between stellar mass and gas-phase metallicity. The MZR for MACS J1115 +01 (Figure 6, left panel) has an offset of 0.2 dex to higher metallicity compared to the local comparison sample at a fixed mass. A double two-sided K-S test yields the probability of stellar mass and metallicity distribution of the cluster members and the local comparison sample to be derived from the same parent population as $28.9 \%$ and $0.03 \%$, respectively, for MACS J1115+01.

The MZR for RX J1532+30 (Figure 6, right panel) follows nearly the same distribution as the SDSS local comparison sample. The median offset in metallicity for RX J1532+30 and the SDSS sample is within the errors of our observations. The $\mathrm{K}-\mathrm{S}$ test probability of stellar mass and metallicity distribution of cluster members and the local comparison sample to be derived from the same parent population is $7.6 \%$ and $28.9 \%$, respectively. This implies that the mass-metallicity distribution of RX J1532+30 and the local comparison sample are subsamples of the same parent population.

We perform a linear fit to derive the MZR relation for our data because the turnover mass in the Zahid et al. (2014) model is poorly constrained for our data due to the stellar mass cut at $\log \left(M_{*} / M_{\odot}\right)<10.52$. The MZR for clusters (Figure 6 , red curve) was fit using the same slope as derived from the SDSS sample (Figure 6, blue curve). The intrinsic scatter in the MZR of the SDSS sample is 0.13 dex. We observe an offset of 0.2 dex between the mean offset in metallicity of MACS J1115 +01 and the local comparison sample (Figure 6, left panel). Student's $t$-test shows that the probability of an offset of 0.2 dex by chance in the metallicity of MACS J1115+01 is less than $1 \%$. In contrast, for RX J1532+01 the mean metallicity offset is only 0.03 dex and the Student's $t$-test probability of a chance offset is $88 \%$, confirming the statistical insignificance of the offset. Thus, the cluster members for MACS J1115+01 show enhanced metallicity at a fixed stellar mass, implying that the cluster environment plays a significant role in driving the chemical evolution of cluster galaxies.

The metallicity offset in MACS $\mathrm{J} 1115+01(0.2$ dex $)$ is significantly higher than the metallicity offset observed in any previous studies of the MZR in the field versus cluster galaxy sample. In contrast, the MZR for RX J1532+30 is consistent with the local comparison sample. Earlier studies of the MZR in cluster versus field galaxy samples constrain the environmental dependence to be around 0.04 dex (Ellison et al. 2009), significantly less than the metallicity offset observed for MACS $\mathrm{J} 1115+01$. However, the cluster galaxy sample for studying the environmental dependence of the MZR is usually collected from a large number of clusters without considering the internal variations among the galaxy clusters. Combining the metallicity data from multiple clusters might be responsible for the nondetection of a metallicity difference between field and cluster galaxies in previous studies. The extremely different MZR for MACS J1115+01 and RX J1532+30 in our observations supports this hypothesis.

\subsection{The Metallicity Gradient in Galaxy Clusters}

We observe an anticorrelation between the cluster member metallicity and projected distance for MACS J1115+01 (Figure 7, left panel). We derive a metallicity gradient derived of $-0.15 \pm 0.08 \mathrm{dex} / \mathrm{Mpc}$ for MACS J1115+01, after a resistant linear regression. The Spearman rank correlation coefficient between metallicity and the projected distance for MACS J1115+01 is $\rho=-0.682$ with a significance of 0.021 . Therefore, the Spearman rank probability of no correlation between the metallicity and the projected distance is $2.1 \%$ for MACS J1115+01. The observed metallicity gradient is unlikely to be driven by the stellar mass of galaxies because we do not observe correlation between stellar mass and projected distance (Spearman's rank of -0.25 , corresponding 


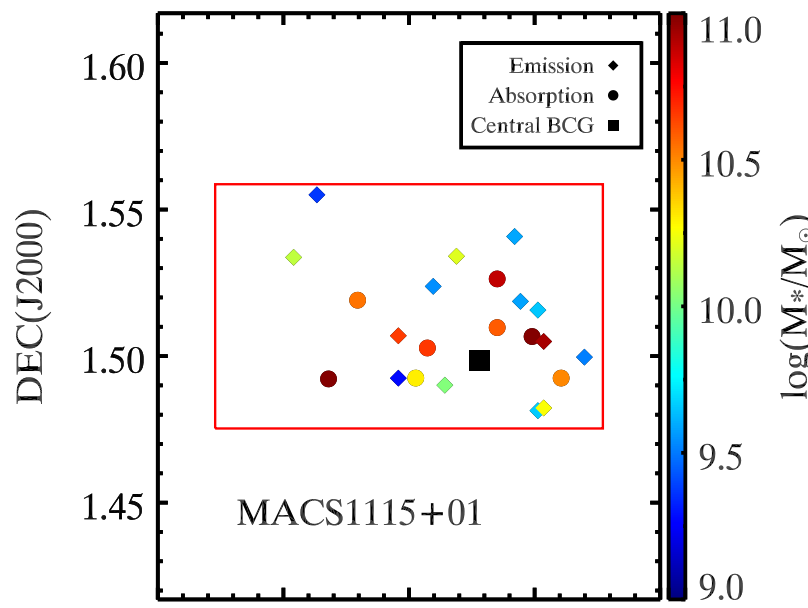

$\begin{array}{lllll}168.74 & 168.83 & 168.92 & 169.01 & 169.10\end{array}$

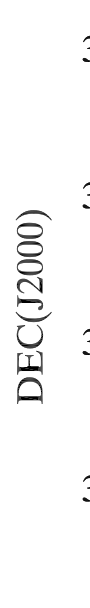

RA(J2000)

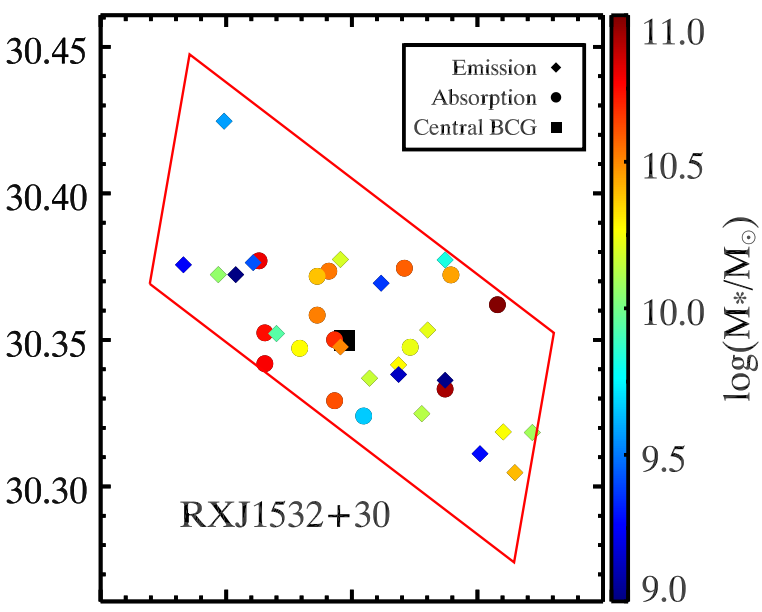

$\begin{array}{lllll}233.05 & 233.14 & 233.23 & 233.32 & 233.41\end{array}$

RA(J2000)

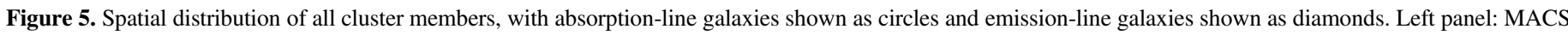

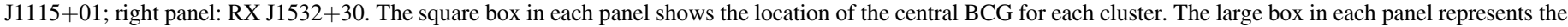

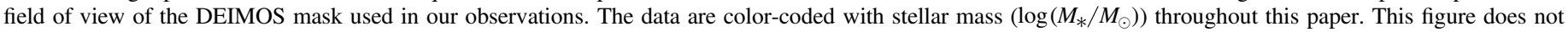
show evidence of mass segregation for both clusters as the spatial location of the cluster member is independent of their stellar mass.

to a significance of $0.8 \sigma$ ). To further disentangle the effect of mass and environment on the metallicity gradient, we analyze the offset in metallicity from the MZR. The metallicity offset also shows a negative correlation with projected distance for MACS J1115+01, albeit within the limitation of our small sample size (see the Appendix).

To quantify the probability of observing a negative abundance gradient due to the intrinsic scatter in the MZR ( 0.13 dex), we bootstrap the data to generate a sample of cluster galaxies with a random stellar mass distribution in the cluster. We assume a Gaussian distribution with a sigma of 0.13 dex for the offset from the MZR. The probability of observing a metallicity gradient of $-0.15 \mathrm{dex} / \mathrm{Mpc}$ caused by the $0.13 \mathrm{dex}$ scatter in the MZR is only $0.1 \%$. Therefore, the observed metallicity gradient is unlikely to be driven by the intrinsic scatter in the MZR.

The metallicity versus projected distance distribution for RX $\mathrm{J} 1532+30$ (Figure 7, right panel) is different from MACS $\mathrm{J} 1115+01$. A slight positive gradient $(0.08 \pm 0.15 \mathrm{dex} / \mathrm{Mpc})$ was derived for RX $\mathrm{J} 1532+30$ using robust linear regression. The Spearman rank correlation coefficient for RX J1532+30 is $\rho=-0.044$ with a significance of 0.87 , i.e., the probability of no correlation between the metallicity and the projected distance is $87 \%$. Thus, the metallicity gradient in RX $\mathrm{J} 1532$ +30 is statistically flat considering all observed cluster members.

Interestingly, the metallicity versus projected distance for RX J1532+30 seems to show two branches. If we exclude the low-mass galaxies $\left(\log \left(M_{*} / M_{\odot}\right)<9.5\right)$, the linear regression for the rest of the cluster members yields a negative metallicity gradient of $-0.14 \pm 0.05 \mathrm{dex} / \mathrm{Mpc}$ (Figure 8, dashed line; hereafter upper branch). Similar linear regression fitting for the low-mass galaxies $\left(\log \left(M_{*} / M_{\odot}\right)>9.5\right)$ yields a positive abundance gradient of $0.3 \pm 0.2 \mathrm{dex} / \mathrm{Mpc}$ (Figure 8 , dotted line; hereafter lower branch). Our observations indicate that the lower-mass galaxies constituting the positive radial gradient are either interlopers or a group of in-plane mergers from a subcluster (further discussed in Section 4.1).

\section{DISCUSSION}

We present the first detections of negative radial gradients in the gas-phase metallicity of galaxies in the cluster environment. We survey two CLASH clusters, MACS J1115+01 and RX $\mathrm{J} 1532+30$, with the DEIMOS/Keck II detecting 22 and 36 cluster members for each, respectively. The gas-phase metallicities are derived for a sample of 12 and 16 star-forming galaxies in MACS J1115+01 and RX J1532+30, respectively, using strong emission-line diagnostics.

\subsection{Gas-phase Metallicity Gradient and Cluster Dynamics}

Even though MACS J1115+01 and RX J1532+30 are both massive clusters with $M_{\text {vir }} \sim 10^{14} M_{\odot} / h$, the observed metallicity distribution is significantly different. The gas-phase metallicities of star-forming galaxies in MACS J1115+01 exhibit an anticorrelation with projected distance, with a gradient of $-0.15 \pm 0.08 \mathrm{dex} / \mathrm{Mpc}$ (Figure 7, left panel), whereas the metallicity distribution of RX J1532+30 with projected distance is suggested to be bimodal (Figure 7, right panel). In addition, the MZR of MACS J1115+01 exhibits an elevated metallicity by $0.2 \mathrm{dex}$ for cluster members as compared to the local SDSS sample (Figure 6, left panel). In contrast, the MZR of RX J1532+30 is statistically consistent with the local comparison sample (Figure 6, right panel).

Contrary to X-ray morphology studies that conclude the relaxed nature of both clusters (Allen et al. 2007), the redshift distribution from our study indicates that the two clusters have different dynamical states. A standard K-S test suggests that the redshift distribution of RX $\mathrm{J} 1532+30$ is non-Gaussian as opposed to the Gaussian redshift distribution of MACS J1115 +01 . The comparison of virial mass estimated from the velocity dispersion measurement of our observations to the virial mass estimated from weak lensing also suggests differences in the dynamical state. From the velocity dispersion, we calculate the virial mass for MACS J1115 to be $M_{\mathrm{vel}}=(1.91 \pm 0.61) \times 10^{15} M_{\odot} / h$, consistent with the lensing mass within the $1 \sigma$ errors $\left(M_{\mathrm{vir}}=(1.13 \pm 0.1)\right.$ 

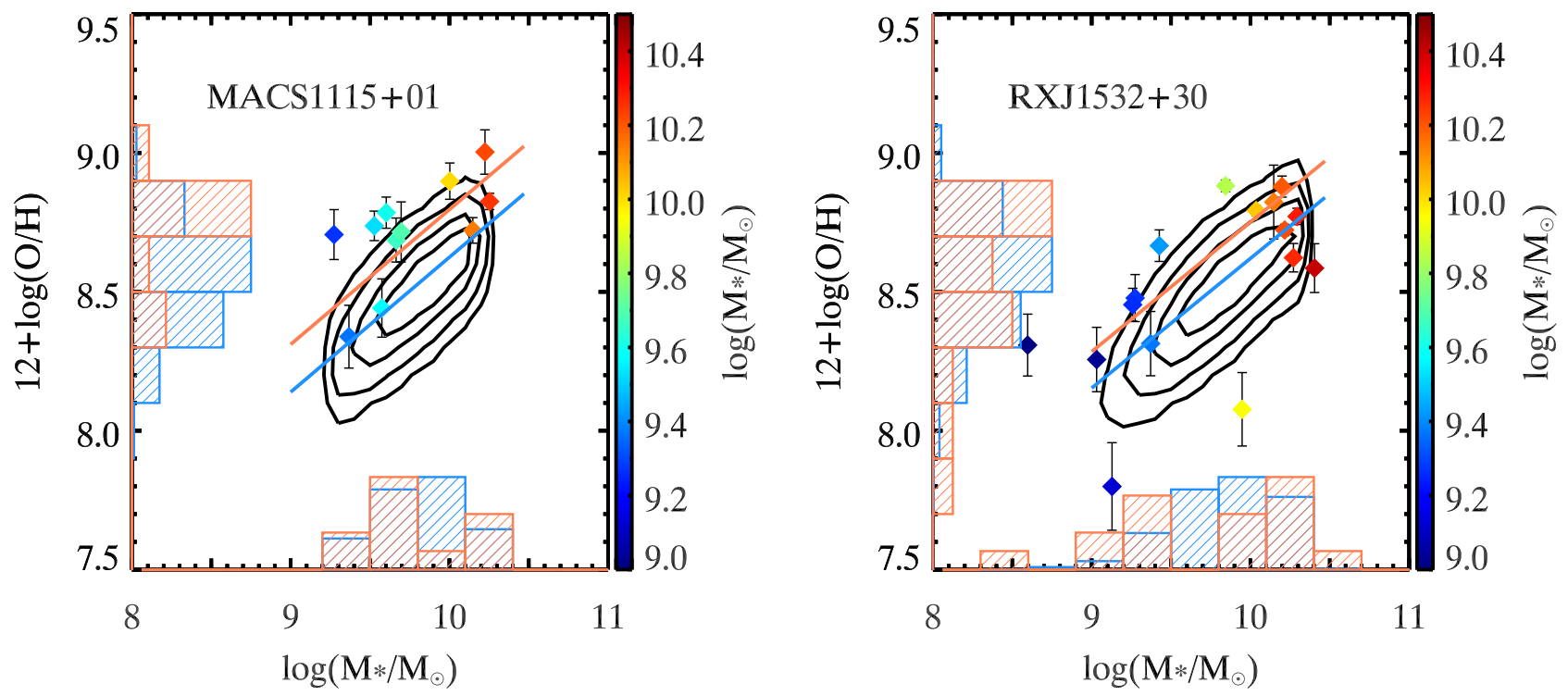

Figure 6. MZR for cluster members. Left panel: MACS J1115+01; right panel: RX J1532+30. The MZR for the local comparison sample is represented as the contours $(25 \%, 50 \%, 70 \%$, and $90 \%)$ in each panel. The red and blue curves represent the best linear fit of the MZR to cluster and SDSS data, respectively. The red and blue histograms in each panel represent the mass and metallicity distribution of the cluster members and SDSS control sample, respectively. The metallicity distributions for MACS J1115+01 and the local comparison sample are clearly different.

$\times 10^{15} M_{\odot} / h$; Merten et al. 2015). For RX J1532, the estimated virial mass is $M_{\text {vel }}=(3.78 \pm 0.73) \times 10^{15} M_{\odot} / h$ from velocity dispersions, about 6 times larger than the lensing mass $\left(M_{\text {vir }}=(0.64 \pm 0.09) \times 10^{15} M_{\odot} / h\right.$; Merten et al. 2015) . In fact, the presence of radio minihalos and the inhomogeneity in large-scale X-ray temperature distributions in RX J1532 also indicate possible unrelaxed dynamics (Hlavacek-Larrondo et al. 2013; Kale et al. 2013).

The two branches in the radial metallicity distribution of RX $\mathrm{J} 1532+30$ could be the result of a minor merger with a cooler subcluster or a group of interloper galaxies. The upper branch $\left(\log \left(M_{*} / M_{\odot}\right)>9.5\right)$ cluster members of RX J1532+30 exhibit a negative abundance gradient with a slope matching the metallicity gradient of MACS J1115+01 (Figures 7 and 8). However, the lower-branch galaxies $\left(\log \left(M_{*} / M_{\odot}\right)<9.5\right)$ exhibit a positive metallicity gradient.

The existence of low-mass galaxies in a separate substructure is confirmed by a virial mass analysis. We measure a velocity dispersion of $773.6 \pm 162.3 \mathrm{~km} \mathrm{~s}^{-1}$ for RX J1532+30 after excluding the lower-branch galaxies, which estimates the virial mass for RX $\mathrm{J} 1532+30$ to be $M_{\mathrm{vel}}=(1.02 \pm 0.55)$ $\times 10^{15} M_{\odot} / h$. The estimated virial mass after excluding the lower-branch galaxies is consistent with the lensing mass within $1 \sigma$ errors, suggesting that the low-mass galaxies belong to a separate substructure and/or are a population of interloper galaxies. The possibility of a minor merger between a cooler subcluster and RX J1532+30 was first suggested by HlavacekLarrondo et al. (2013) based on the spatial asymmetry in the large-scale X-ray temperature of RX J1532+30. We hypothesize that the upper-branch galaxies $\left(\log \left(M_{*} / M_{\odot}\right)>9.5\right)$ belong to the dynamically relaxed massive component of the cluster RX J1532+30, with a metallicity gradient similar to the dynamically relaxed cluster MACS J1115+01. The lowerbranch galaxies belong to a merging cooler subcluster and/or interloper galaxies that are misidentified as cluster galaxies due to projection effects. To understand whether projection effects play a role in our metallicity gradient measurements, we compare our results with the metallicity gradient from Rhapsody-G simulations.

\subsection{Comparison with Rhapsody-G Simulations}

We compare our data to the RHAPSODY-G simulations performed with the RAMSES (Teyssier 2002) adaptive mesh refinement (AMR) code (Hahn et al. 2015; Martizzi et al. 2015; $\mathrm{Wu}$ et al. 2015) because the mass range of simulated clusters matches the mass of observed clusters. The RHAPSODY-G sample currently includes cosmological hydrodynamical zoomin simulations of 10 massive galaxy clusters of virial mass $M_{\text {vir }} \sim 6 \times 10^{14} M_{\odot} / h$. The simulations assume standard $\Lambda \mathrm{CDM}$ cosmology with $\Omega_{M}=0.25, \Omega_{\Lambda}=0.75, \Omega_{\mathrm{b}}=0.045$, and $h=0.7$. The different cosmological parameters of the observations and simulations will introduce an offset in distance measurement up to $20 \mathrm{kpc}$. In the RHAPSODY-G terminology, the simulations used for our comparison have "R4K resolution": dark matter particle mass of $m_{\mathrm{dm}}=8.22 \times 10^{8} h^{-1} M_{\odot}$, initial baryonic matter resolution element of $m_{\mathrm{b}}=m_{\mathrm{dm}} \Omega_{\mathrm{b}} /\left(\Omega_{M}-\Omega_{\mathrm{b}}\right)=1.80 \times 10^{8} h^{-1} M_{\odot}$, and maximum resolution reached in these simulations of $\Delta x=3.8 h^{-1} \mathrm{kpc}$ (in physical units). Gas cooling, star formation, metal enrichment from supernovae, supernova feedback, and AGN feedback are included in RHAPSODY-G.

These simulations successfully reproduce the mass distribution, baryonic abundances, cool-core/non-cool-core dichotomy, and SZ properties of observed clusters (Hahn et al. 2015). We select simulated cluster member galaxies with star formation rate greater than $1 M_{\odot} \mathrm{yr}^{-1}$ to best compare with the observations of metallicity gradient. Note that the absolute metallicity of the simulations is lower than that of observations; therefore we cannot directly compare the absolute simulated and observed metallicities. This offset may be related to the observational metallicity calibration used (e.g., Kewley \& Ellison 2008). We do not compare the impact of environment on the MZR in simulations because there are no field galaxies in the simulations. However, we can investigate signatures of 

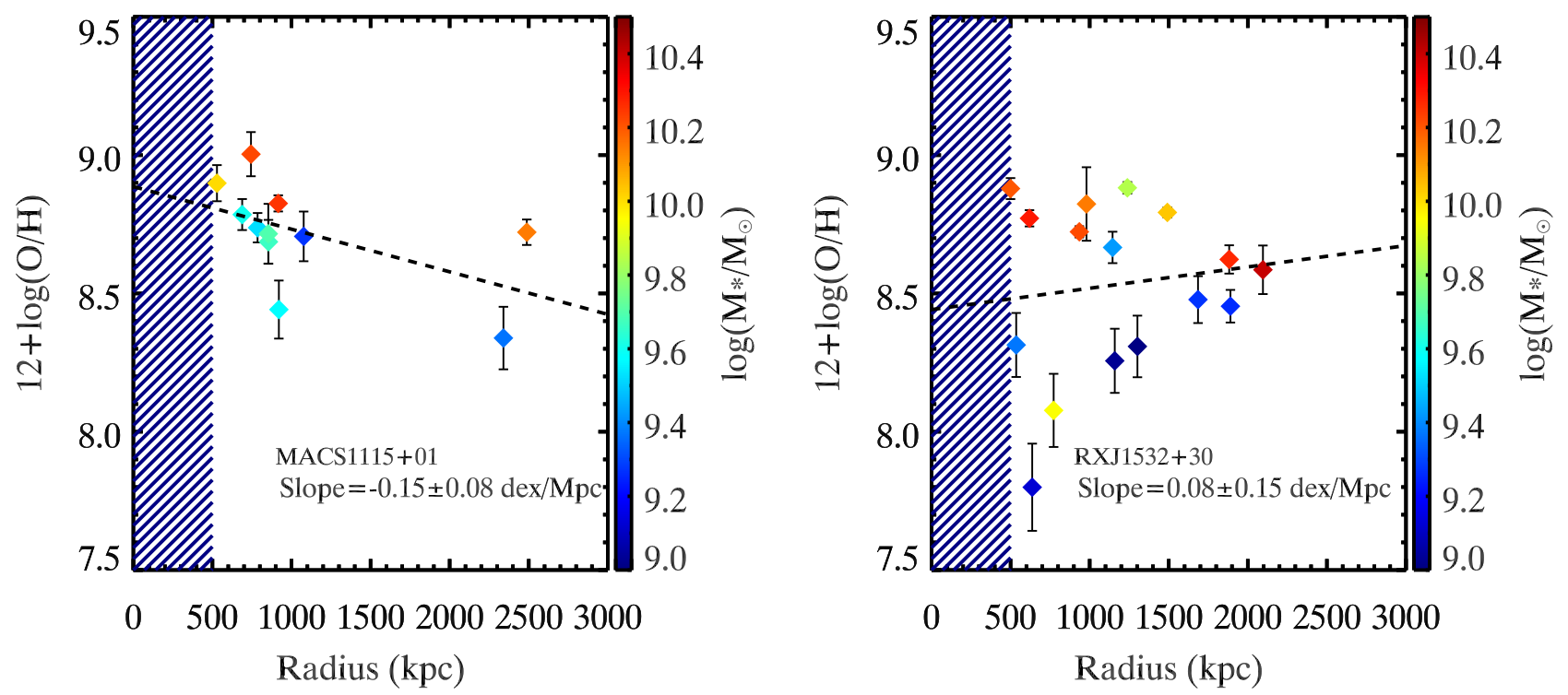

Figure 7. Radial metallicity distribution for individual clusters, where metallicity is derived using the N2S2 method described in Section 2.10. Left panel: MACS $\mathrm{J} 1115+01$; right panel: RX J1532+30. The dashed line in each figure is the best linear fit to the data. The slope and standard error in slope of the best-fitting line are labeled in each figure. The metallicity of cluster members in MACS J1115+01 anticorrelates with the projected distance, whereas the radial metallicity distribution of RX J1532+30 is bimodal, which is collectively equivalent to a flat metallicity gradient. Our GLOW-AO survey is biased against the observation of cluster member galaxies in the central part of the cluster; therefore, we have shaded the central $500 \mathrm{kpc}$ of the cluster (Section 2.3).

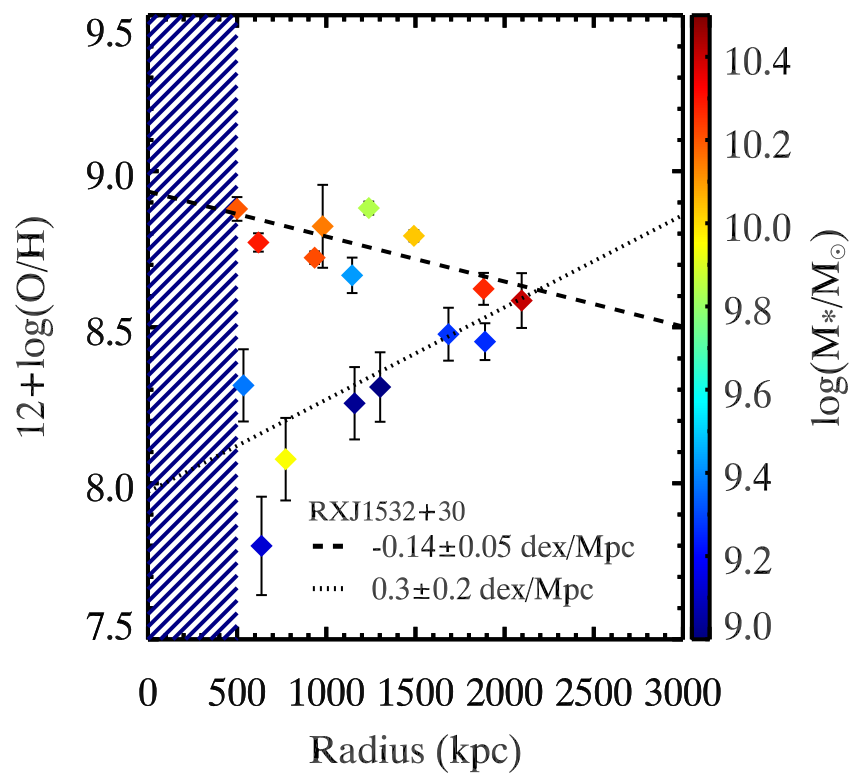

Figure 8. Bimodal metallicity distribution of RX $\mathrm{J} 1532+30$ using a stellar mass cut of $\log \left(M_{*} / M_{\odot}\right)=9.5$. The dashed line represents the best-fitting metallicity gradient line for galaxies with $\log \left(M_{*} / M_{\odot}\right)>9.5$ (upper branch), and the dotted line gives the best-fitting metallicity gradient line for galaxies with $\log \left(M_{*} / M_{\odot}\right)<9.5$ (lower branch). The figure shows a negative abundance gradient for high-mass galaxies with slope, similar to MACS J1115+01 (Figure 7, left panel), whereas low-mass galaxies show a positive abundance gradient.

relative metallicities, such as metallicity gradients, in the Rhapsody-G simulations. We note that a direct comparison with the simulations of Taylor \& Kobayashi (2015), who use a sophisticated supernova feedback and chemical evolution prescription, was not possible because their current simulations do not contain relaxed clusters on the mass scales of interest here.

We select the simulated galaxy cluster, which has the closest value of concentration to the observations. The concentration is a proxy for the age and dynamical state of the cluster (Dutton \& Macciò 2014). Therefore, we use the concentration parameter of the simulations $\left(c_{\mathrm{vir}}=r_{\mathrm{vir}} / r_{s}\right.$, where $r_{\mathrm{vir}}$ is the virial radial and $r_{s}$ is the scale radius of the density profile of the cluster) to match with the observations. Both MACS J1115+01 and RX $\mathrm{J} 1532+30$ have similar concentration parameters, resulting in the selection of the halo-377 for both clusters. Observationally, the similar concentration of MACS J1115+01 and RX J1532 +30 does not fully rule out the dynamically unrelaxed nature of $\mathrm{RX} \mathrm{J1532+30}$ because concentration is not strongly affected by minor merger events.

The comparison simulated cluster exhibits a negative abundance gradient of $-0.04 \pm 0.03 \mathrm{dex} / \mathrm{Mpc}$ (Figure 9). The negative metallicity gradient in the simulation is not driven by the stellar mass in accordance with the mass-independent abundance gradient of our observations. The existence of a negative metallicity gradient in simulations confirms that the observed metallicity gradient is not a projection effect, but the shallower slope may be related to a lack of information about the projection axis of the observed clusters. Our future work will include a comprehensive analysis of the projection effects on the observed metallicity gradient using simulations (Gupta et al. 2016).

\subsection{Origin of Cluster-scale Metallicity Gradient 4.3.1. Truncation of Galactic Disk}

A galaxy moving through the dense environment of the galaxy cluster experiences galaxy harassment and ram pressure stripping, resulting in truncation of the outer galactic disk. Although the metallicity measurements via strong emission lines are biased toward the high surface brightness regions, the truncation of the outer galactic disk can enhance the bias in metallicity measurements to the inner metal-rich galactic disk (e.g., Yuan et al. 2013; Genel 2016). Therefore, as the galaxy falls into the galaxy cluster, a consecutive decrease in the galactic disk size as a result of the loss of the outer disk due to 


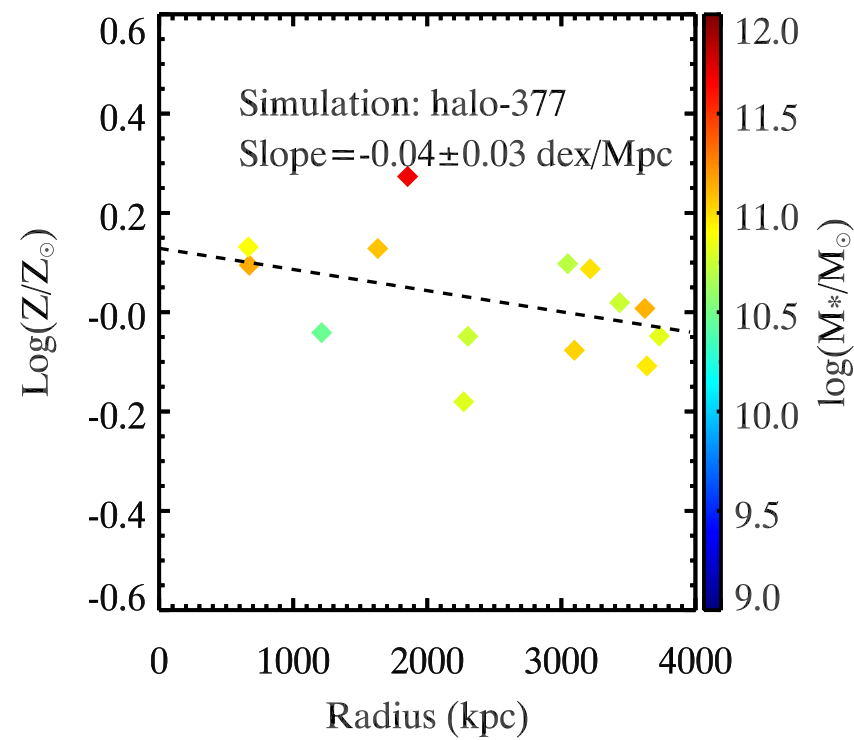

Figure 9. Radial distribution of star-forming galaxies in halo-377 in the Rhapsody-G simulations. We have used projected distance instead of radial distance and color-coded the simulated galaxies according to their stellar mass for a direct comparison with the observations. The simulations show a massindependent negative metallicity gradient similar to the metallicity gradient in our observations.

ram pressure stripping or galaxy harassment would result in successive high-metallicity measurements in spatially nonresolved observations. This effect might play a role in causing the observed negative abundance gradient in the global metallicity of star-forming cluster members.

Morphological changes in the galaxy as a function of radial distance are a direct consequence of the galactic disk destabilization/truncation due to galaxy harassment and/or ram pressure stripping. Unfortunately, the existing imaging data from CFHT and SDSS are not deep enough to observe these morphological changes, and HST data only span the inner $\sim 300 \mathrm{kpc}$ of the cluster. Thus, we require deeper imaging data in the outskirts of galaxy clusters to observe the morphological changes in star-forming galaxies with radial distance. Separating the effect of ram pressure and galaxy harassment is impossible with the current sample, as both galaxy clusters in our current sample are X-ray luminous. Future metallicity gradient observations with a broader sample of X-ray-luminous (ram pressure dominant) and the X-ray nonluminous (galaxy harassment dominant) clusters can help to distinguish the relative role of ram pressure stripping and galaxy harassment. Finally, in order to understand the effect of disk truncation on metallicity gradients, deep imaging and spatially resolved spectroscopic observations are required.

\subsubsection{Self-enrichment via Strangulation}

In addition to disk truncation due to ram pressure stripping or galaxy harassment, the ICM also strips the galaxy of the loosely bound hot/warm halo gas (fuel for future star formation). Depriving the galaxy of a fresh supply of gas results in a gradual decline in star formation as the galaxy consumes its existing cold gas reservoir (strangulation; Larson et al. 1980). The dilution from the inflowing pristine gas in the strangulation mode does not exist, which results in selfenrichment of the galaxy as it goes through successive cycles of star formation. Assuming that the infall timescale for galaxies in the inner region of the cluster is greater than the infall timescale for galaxies in the outskirts, the star-forming galaxies in the inner regions, which have undergone more cycles of star formation, would be more metal-rich compared to galaxies in the outskirts. Thus, preferential self-enrichment of galaxies in the inner regions of the cluster as compared to the galaxies in the outskirts can also generate the observed negative abundance gradient. In order to study the self-enrichment of galaxies in galaxy clusters due to strangulation, we require integral field spectroscopy of a mass-matched sample of starforming cluster galaxies at different cluster-centric distances.

The stellar mass of a galaxy will also increase during selfenrichment via strangulation (Peng et al. 2015). Therefore, preferential self-enrichment of galaxies in the inner regions of the cluster as compared to the galaxies in the outskirts should result in a direct correlation between the stellar mass and cluster-centric distance. The lack of an apparent stellar mass segregation for emission-line galaxies might be a result of one or more of the following: (i) selective removal of massive quenched galaxies due to our sample bias toward star-forming galaxies; (ii) mass growth as a function of infall time depends on the details of star formation history, such as mergers and ram-pressure-enhanced star formation rate (Dressler \& Gunn 1983; Gavazzi et al. 1995); (iii) projection effects lead to uncertainty in radial distance measurement (discussed in the subsequent subsection).

\subsubsection{Systematic Uncertainties Due to Projection Effects}

Observational measurements of the absolute cluster-centric distance are impossible; we only have information about the projected distance of a galaxy in a triaxial cluster halo. Adding the line-of-sight velocity of the galaxy relative to the galaxy cluster can reduce the uncertainty in the absolute cluster-centric distance measurement. A recent study by Maier et al. (2016) uses a projected phase-space diagram to separate the cluster members into groups of accreted versus infalling galaxies. Adding the line-of-sight velocity via the projected phase-space diagram does not improve the signature of mass segregation for star-forming galaxies in our observations (Figure 10). In addition, the low-mass cluster members for RX J1532+30 (lower branch on Figure 8) do not occupy any special location on the projected phase-space diagram (Figure 10, right panel), which we propose belongs to an in-plane merging cooler subcluster and/or interloper galaxies. However, even the projected phase-space diagram does not contain full threedimensional information about a galaxy cluster, and therefore it is insufficient to rule out the uncertainty in radial distance measurements.

Our current simulations do not have sufficient resolution to fully investigate the effects of disk truncation and selfenrichment on the origin of the metallicity gradient. Highresolution simulations would allow us to understand the change in a galaxy's properties, such as metallicity, stellar mass, and fraction of cold and hot gas, as the galaxy falls into the cluster environment. The existence of resolved low stellar mass galaxies in the high-resolution simulations would provide a better comparison with the observations. A more detailed analysis of galaxy tracking in simulations with high resolution is required to properly study the effects influencing the metallicity distribution in cluster satellites. Moreover, to fully understand the origin of cluster-scale metallicity gradients, we 

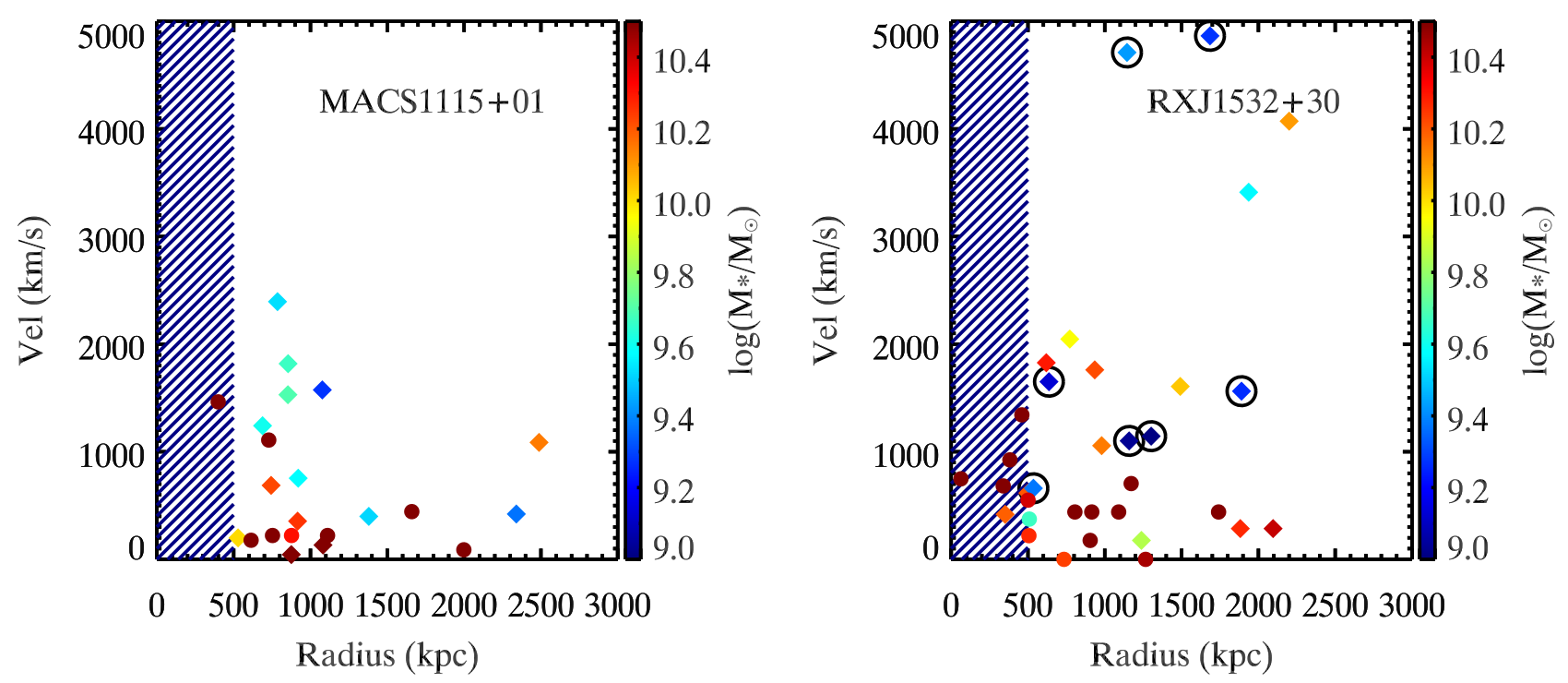

Figure 10. Projected phase-space diagram for the observed clusters. Left panel: MACS J1115+01; right panel: RX J1532+30. Emission-line galaxies are represented as diamonds and absorption-line galaxies as circles. The data are color-coded with stellar mass. There is inconclusive evidence of mass segregation from the projected phase-space diagram for the two clusters. The open circles in the right panel represent galaxies that belong to the $\operatorname{lower}$ branch $\left(\log \left(M_{*} / M_{\odot}\right)<9.5\right)$ in Figure 8 . The random positions of the merging subcluster galaxies suggest an in-plane merger.

require metallicity gradient observations with a broader sample of a range of X-ray luminosity and dynamical state.

\section{CONCLUSIONS}

Using the multi-object optical spectrograph DEIMOS on the Keck II telescope, we observe two CLASH clusters at $z \sim 0.35$, MACS J1115+01 and RX J1532+30. We analyze the gasphase metallicity of star-forming galaxies derived from [N II]/ $[\mathrm{S} \mathrm{II}]$ and $[\mathrm{N} \mathrm{II}] / \mathrm{H} \alpha$, as a function of projected distance from the central BCG. Our main results are as follows:

1. For MACS J1115+01, we identify 22 (14-emission-line galaxies) cluster members corresponding to a velocity dispersion of $\sigma_{\mathrm{cl}}=960 \pm 147 \mathrm{~km} \mathrm{~s}^{-1}$. For RX J1532 +30 , we identify 36 (21-emission-line galaxies) cluster members corresponding to a velocity dispersion of $\sigma_{\mathrm{cl}}=1487 \pm 213 \mathrm{~km} \mathrm{~s}^{-1}$. The virial mass estimated from the velocity dispersion is consistent with the gravitational lensing mass for MACS J1115+01, but is six times more than the gravitational lensing mass for RX $\mathrm{J} 1532+30$.

2. We observe a significant metallicity enhancement in the star-forming galaxies in MACS J1115+01 (0.2 dex) at a fixed stellar mass range with respect to the local comparison galaxies. This is the largest metallicity enhancement observed for galaxy clusters to date. The MZR of RX J1532+30 is consistent with the MZR of local comparison galaxies. The significant difference between the MZRs of MACS J1115+01 and RX J1532 +30 shows that the nondetection of metallicity enhancement in galaxy clusters in previous studies might be due to the combination of data from multiple clusters with different MZR.

3. We present the first observation of a negative abundance gradient in the global metallicity of star-forming galaxies in galaxy clusters. We observe an abundance gradient of $-0.15 \pm 0.08 \mathrm{dex} / \mathrm{Mpc}$ for MACS J1115+01 and a bimodal-like metallicity distribution for RX $\mathrm{J} 1532+30$.
The upper-branch galaxies in RX J1532+30 also show a negative abundance gradient similar to MACS J1115+01 $(-0.14 \pm 0.05 \mathrm{dex} / \mathrm{Mpc})$. We speculate that the lowerbranch galaxies in RX $\mathrm{J} 1532+30$ belong to a merging subcluster and/or are interloper galaxies. The velocity dispersion after excluding the lower-branch galaxies is $773.6 \pm 162.3 \mathrm{~km} \mathrm{~s}^{-1}$, which gives a virial mass consistent with the lensing mass, suggesting a separate substructure for the lower-branch galaxies.

4. Star-forming galaxies in a simulated galaxy cluster also exhibit a negative abundance gradient, but with a shallower slope $(-0.04 \pm 0.03 \mathrm{dex} / \mathrm{Mpc})$.

5 . We speculate that the origin of the negative abundance gradient in galaxy clusters could be due to (i) the truncation of an outer galactic disk due to galaxy harassment or ram pressure stripping and (ii) the selfenrichment of the galaxy after the ICM cuts off the gas supply for star formation.

6. We hypothesize that a cluster-scale gradient in the ISM metallicity of galaxy members exists in clusters that (i) are X-ray luminous (i.e., have enough hot ICM) and (ii) are in a relatively relaxed dynamical state (i.e., no significant recent merger).

In our follow-up work, we will use the Rhapsody-G simulations at significantly higher resolution to investigate the origin of metallicity gradients in galaxy clusters. To fully test our hypothesis, we require deep imaging and integral field spectroscopy for galaxy clusters with different X-ray and dynamical properties.

The authors thank the referee for the useful and comprehensive referee report. This work is based on data obtained at the W. M. Keck Observatory. We are grateful to the Keck Observatory staff for assistance with our observations, especially Jim Lyke, Marc Kassis, and Luca Rizzi. The Observatory was made possible by the generous financial support of the W. M. Keck Foundation. A.G. acknowledges Prof. Micheal Cooper for his help with spec2d pipeline, I-Ting 

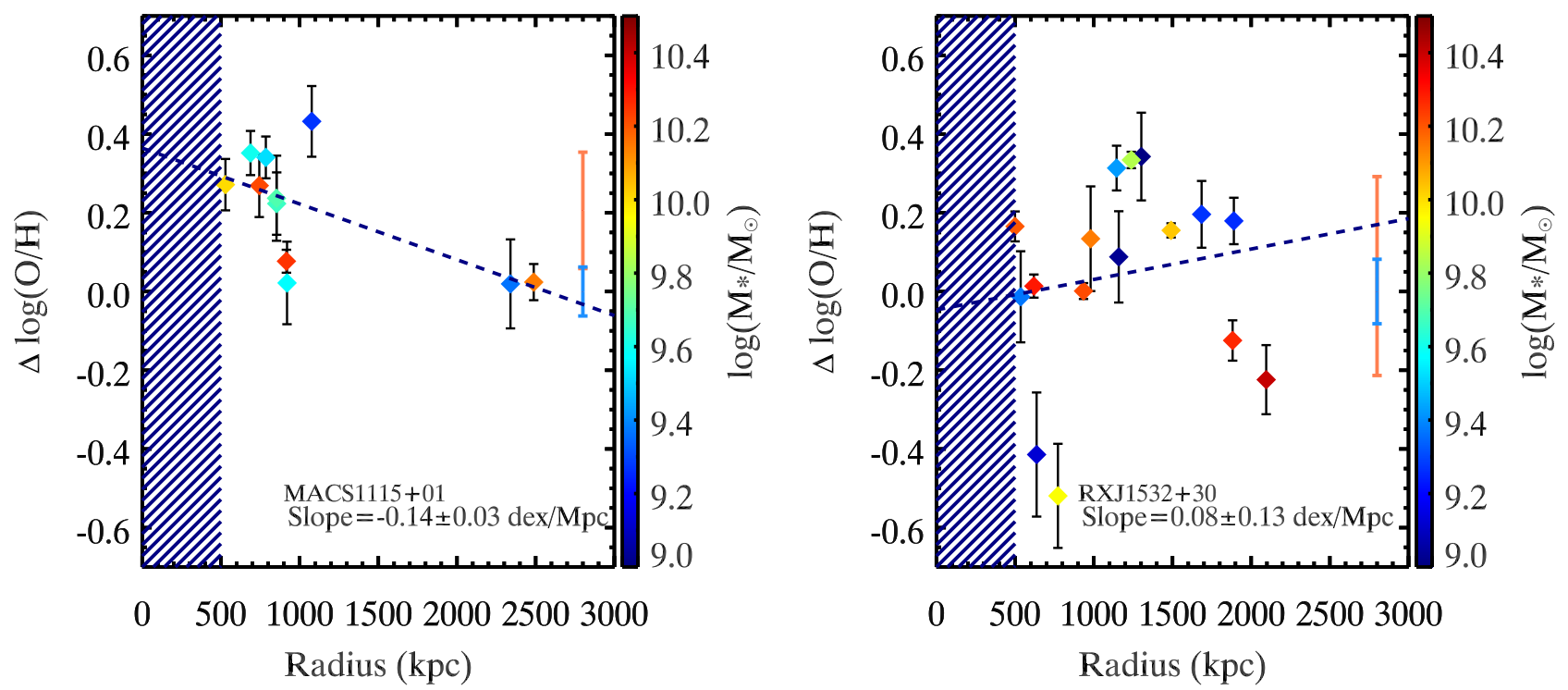

Figure 11. Offset from the MZR of the local comparison sample (Figure 6, red curve) with the projected distance. Left panel: MACS J1115+01; right panel: RX $\mathrm{J} 1532+30$. The dashed line represents the best linear fit. The slope and standard error in slope are labeled in each figure. The blue and red error bars represent the scatter and median metallicity offset of the comparison sample and the cluster data. The offset from the MZR also exhibits anticorrelation with the projected distance for MACS J1115+01, which suggests that the metallicity enhancement toward the cluster center is not driven by the stellar mass.

Ho for supplying the SDSS data, and Melanie Kaasinen for her help in the editing of this paper. We thank C.J. Ma and Harald Ebeling for sharing their CFHT color images for the cluster RX $\mathrm{J} 1532+30$. L.J.K. gratefully acknowledges the support of an ARC Future Fellowship and ARC Discovery Project DP130103925. K.-V.H.T. acknowledges support by the National Science Foundation under grant no. 1410728. P.T. acknowledges support from a Discovery Project DP150104329 from the Australian Research Council. The authors wish to recognize and acknowledge the very significant cultural role and reverence that the summit of Mauna Kea has always had within the indigenous Hawaiian community. We are most fortunate to have the opportunity to conduct observations from this mountain.

\section{APPENDIX \\ THE OFFSET FROM THE MZR}

To separate the effect of mass and environment on the chemical evolution, we study the offset in cluster member metallicity from the MZR of the local comparison sample. For MACS J1115+01, cluster members in the inner parts show enhanced metallicity at a fixed stellar mass range compared to cluster members in the outskirts (Figure 11, left panel). In contrast, cluster members in RX $\mathrm{J} 1532+30$ do not show a correlation between the metallicity offset and the projected distance (Figure 11, right panel). The existence of the negative gradient in the offset from the MZR for MACS J1115+01 suggests that the cluster-scale negative abundance gradient is not driven by the stellar mass of galaxies. However, our conclusion is limited by the small sample size of our data.

\section{REFERENCES}

Abazajian, K. N., Adelman-McCarthy, J. K., Agüeros, M. A., et al. 2009, ApJS, 182, 543

Adami, C., Biviano, A., Durret, F., \& Mazure, A. 2005, A\&A, 443, 17

Alatalo, K., Cales, S. L., Rich, J. A., et al. 2016, ApJS, 224, 38
Allen, S. W., Rapetti, D. A., Schmidt, R. W., et al. 2007, MNRAS, 383, 879 Arnouts, S., Cristiani, S., Moscardini, L., et al. 1999, MNRAS, 310, 540 Baldwin, A., Phillips, M. M., \& Terlevich, R. 1981, PASP, 93, 817

Balogh, M. L., Navarro, J. F., \& Morris, S. L. 2000, ApJ, 540, 113

Bialas, D., Lisker, T., Olczak, C., Spurzem, R., \& Kotulla, R. 2015, A\&A, 576, A103

Brinchmann, J., Charlot, S., White, S. D. M., et al. 2004, MNRAS, 351, 1151 Bruzual, G., \& Charlot, S. 2003, MNRAS, 344, 1000

Calzetti, D., Armus, L., Bohlin, R. C., et al. 2000, ApJ, 533, 682

Cappellari, M., \& Emsellem, E. 2004, PASP, 116, 138

Chabrier, G. 2003, PASP, 115, 763

Cooper, M. C., Aird, J. A., Coil, A. L., et al. 2011, ApJS, 193, 14

Cooper, M. C., Tremonti, C. A., Newman, J. A., \& Zabludoff, A. I. 2008, MNRAS, 390, 245

Cora, S. A., Tornatore, L., Tozzi, P., \& Dolag, K. 2008, MNRAS, 386, 96

Darvish, B., Mobasher, B., Sobral, D., et al. 2015, ApJ, 814, 84

Davé, R., Finlator, K., \& Oppenheimer, B. D. 2011, MNRAS, 416, 1354

Davis, M., Meiksin, A., Strauss, M. A., da Costa, L. N., \& Yahil, A. 1988, ApJL, 333, L9

De Grandi, S., \& Molendi, S. 2001, ApJ, 551, 153

De Lucia, G., \& Blaizot, J. 2007, MNRAS, 375, 2

Dopita, M. A., Kewley, L. J., Sutherland, R. S., \& Nicholls, D. C. 2016, Ap\&SS, 361, 61

Dressler, A. 1980, ApJ, 236, 351

Dressler, A., \& Gunn, J. E. 1983, ApJ, 270, 7

Dressler, A., \& Shectman, S. A. 1988, AJ, 95, 985

Dutton, A. A., \& Macciò, A. V. 2014, MNRAS, 441, 3359

Ebeling, H., Edge, A. C., Böhringer, H., et al. 1998, MNRAS, 301, 881

Ebeling, H., Edge, A. C., Mantz, A., et al. 2010, MNRAS, 407, 83

Ellison, S. L., Simard, L., Cowan, N. B., et al. 2009, MNRAS, 396, 1257

Faber, S. M., Phillips, A. C., Kibrick, R. I., et al. 2003, Proc. SPIE, 4841, 1657

Gavazzi, G., Randone, I., \& Branchini, E. 1995, ApJ, 438, 590

Genel, S. 2016, ApJ, 822, 107

Gunn, J. E., Gott, J., \& Richard, I. 1972, ApJ, 176, 1

Gupta, A., Yuan, T.-T., Kassine, M., \& Kewley, L. J. 2016, PASP, submitted

Hahn, O., Martizzi, D., Wu, H.-Y., et al. 2015, MNRAS, submitted (arXiv:1509.04289)

Hausman, M. A., \& Ostriker, J. P. 1978, ApJ, 224, 320

Hinshaw, G., Larson, D., Komatsu, E., et al. 2013, ApJS, 208, 19

Hlavacek-Larrondo, J., Allen, S. W., Taylor, G. B., et al. 2013, ApJ, 777, 163

Houghton, R. C. W. 2015, MNRAS, 451, 3427

Hughes, T. M., Cortese, L., Boselli, A., Gavazzi, G., \& Davies, J. I. 2013, A\&A, 550, A115

Huss, A., Jain, B., \& Steinmetz, M. 1999, ApJ, 517, 64

Ilbert, O., Arnouts, S., McCracken, H. J., et al. 2006, A\&A, 457, 841 
Kacprzak, G. G., Yuan, T., Nanayakkara, T., et al. 2015, ApJL, 802, L26

Kale, R., Venturi, T., Giacintucci, S., et al. 2013, A\&A, 557, A99

Kapferer, W., Kronberger, T., Weratschnig, J., et al. 2007, A\&A, 466, 813

Kauffmann, G., Heckman, T. M., Tremonti, C., et al. 2003a, MNRAS, 346,1055

Kauffmann, G., Heckman, T. M., White, S. D. M., et al. 2003b, MNRAS, 341, 54

Kewley, L. J., Dopita, M. A., Sutherland, R. S., Heisler, C. A., \& Trevena, J. 2001, ApJ, 556, 121

Kewley, L. J., \& Ellison, S. L. 2008, ApJ, 681, 1183

Kewley, L. J., Groves, B., Kauffmann, G., \& Heckman, T. 2006, MNRAS, 372,961

Kewley, L. J., Jansen, R. A., \& Geller, M. J. 2005, PASP, 117, 227

Kewley, L. J., Maier, C., Yabe, K., et al. 2013, ApJL, 774, L10

Larson, R. B., Tinsley, B. M., \& Caldwell, C. N. 1980, ApJ, 237, 692

Maier, C., Kuchner, U., Ziegler, B. L., et al. 2016, A\&A, 590, A108

Martínez, H. J., Coenda, V., \& Muriel, H. 2008, MNRAS, 391, 585

Martizzi, D., Hahn, O., Wu, H.-Y., et al. 2016, MNRAS, 459, 4408

Merten, J., Meneghetti, M., Postman, M., et al. 2015, ApJ, 806, 4

Moore, B., Katz, N., Lake, G., Dressler, A., \& Oemler, A. 1996, Natur, 379,613

Mouhcine, M., Baldry, I. K., \& Bamford, S. P. 2007, MNRAS, 382, 801

Navarro, J. F., Frenk, C. S., \& White, S. D. M. 1997, ApJ, 490, 493

Osterbrock, D. E. 1989, Astrophysics of Gaseous Nebulae and Active Galactic Nuclei (Mill Valley, CA: Univ. Science Books)

Ostriker, J. P., \& Tremaine, S. D. 1975, ApJL, 202, L113

Pasquali, A., Gallazzi, A., \& van den Bosch, F. C. 2012, MNRAS, 425, 273

Peng, Y., Maiolino, R., \& Cochrane, R. 2015, Natur, 521, 192
Peng, Y.-J., \& Maiolino, R. 2014, MNRAS, 438, 262

Pettini, M., \& Pagel, B. E. J. 2004, MNRAS, 348, L59

Phillips, M. M., Jenkins, C. R., Dopita, M. A., Sadler, E. M., \& Binette, L. 1986, AJ, 91, 1062

Postman, M., Coe, D., Benítez, N., et al. 2012, ApJS, 199, 25

Rasia, E., Borgani, S., Murante, G., et al. 2015, ApJL, 813, L17

Rauch, T., Werner, K., Bohlin, R., \& Kruk, J. W. 2013, A\&A, 560, A106

Ribeiro, A., Lopes, P., \& Rembold, S. 2013, A\&A, 556, A74

Rich, J. A., Kewley, L. J., \& Dopita, M. A. 2011, ApJ, 734, 87

Sarzi, M., Shields, J. C., Schawinski, K., et al. 2010, MNRAS, 402, 2187

Scudder, J. M., Ellison, S. L., \& Mendel, J. T. 2012, MNRAS, 423, 2690

Serna, A., \& Gerbal, D. 1996, A\&A, 309, 65

Shimakawa, R., Kodama, T., Steidel, C. C., et al. 2015, MNRAS, 451, 1284

Taylor, P., \& Kobayashi, C. 2015, MNRAS, 448, 1835

Teyssier, R. 2002, A\&A, 385, 337

Tran, K.-V. H., Nanayakkara, T., Yuan, T., et al. 2015, ApJ, 811, 28

Valentino, F., Daddi, E., Strazzullo, V., et al. 2015, ApJ, 801, 132

Vazdekis, A., Sánchez-Blázquez, P., Falcón-Barroso, J., et al. 2010, MNRAS, 404, 1639

Veilleux, S., \& Osterbrock, D. E. 1987, ApJS, 63, 295

Wu, H.-Y., Evrard, A. E., Hahn, O., et al. 2015, MNRAS, 452, 1982

Yahil, A., \& Vidal, N. V. 1977, ApJ, 214, 347

Yan, R., \& Blanton, M. R. 2012, ApJ, 747, 61

York, D. G., Adelman, J., Anderson, J. E., et al. 2000, AJ, 120, 1579

Yuan, T.-T., Kewley, L. J., \& Rich, J. 2013, ApJ, 767, 106

Zahid, H. J., Dima, G. I., Kudritzki, R.-P., et al. 2014, ApJ, 791, 130

Zandivarez, A., \& Martínez, H. J. 2011, MNRAS, 415, 2553 